BULLETIN (New Series) OF THE

AMERICAN MATHEMATICAL SOCIETY

Volume 40, Number 3, Pages 281-321

S 0273-0979(03)00985-6

Article electronically published on April 8, 2003

\title{
MEASURES AND DIMENSIONS IN CONFORMAL DYNAMICS
}

\author{
MARIUSZ URBAŃSKI
}

\begin{abstract}
This survey collects basic results concerning fractal and ergodic properties of Julia sets of rational functions of the Riemann sphere. Frequently these results are compared with their counterparts in the theory of Kleinian groups, and this enlarges the famous Sullivan dictionary. The topics concerning Hausdorff and packing measures and dimensions are given most attention. Then, conformal measures are constructed and their relations with Hausdorff and packing measures are discussed throughout the entire article. Also invariant measures absolutely continuous with respect to conformal measures are touched on. While the survey begins with facts concerning all rational functions, much time is devoted toward presenting the well-developed theory of hyperbolic and parabolic maps, and in Section 3 the class NCP is dealt with. This class consists of such rational functions $f$ that all critical points of $f$ which are contained in the Julia set of $f$ are non-recurrent. The NCP class comprises in particular hyperbolic, parabolic and subhyperbolic maps. Our last section collects some recent results about other subclasses of rational functions, e.g. Collet-Eckmann maps and Fibonacci maps. At the end of this article two appendices are included which are only loosely related to Sections 1-4. They contain a short description of tame mappings and the theory of equilibrium states and Perron-Frobenius operators associated with Hölder continuous potentials.
\end{abstract}

\section{Dimensions of Julia Sets}

A first issue we will be dealing with in this article is to describe various fractals of Julia sets as captured by the Hausdorff, packing and box dimensions. Afterwards, we address the question of when the corresponding Hausdorff and packing measures are positive and finite. Given a subset $A$ of a metric space $(X, d)$ a countable family $\left\{B\left(x_{i}, r_{i}\right)\right\}_{i=1}^{\infty}$ of open balls centered at points of $A$ is said to be a packing of $A$ if and only if for any pair $i \neq j$

$$
d\left(x_{i}, x_{j}\right) \geq r_{i}+r_{j} .
$$

The supremum $\sup \left\{r_{i}: i \geq 1\right\}$ is called the radius of the packing $\left\{B\left(x_{i}, r_{i}\right)\right\}_{i=1}^{\infty}$. Given in addition a positive radius $r>0$, denote by $N(A, r)$ the minimal number of open balls with radius $r$ needed to cover $A$ and by $P(A, r)$ the maximal number of open balls with radius $r$ forming a packing of $A$.

In order to get an idea of what the box dimension is, imagine a two-dimensional smooth surface $A$ in the Euclidean space $\mathbb{R}^{3}$. It is reasonable to expect the minimal number $N(A, r)$ and the maximal number $P(A, r)$ to be some multiples of $r^{-2}$. The

Received by the editors December 22, 1999, and, in revised form, January 8, 2003.

2000 Mathematics Subject Classification. Primary 35F35, 37D35; Secondary 37F15, 37D20, 37D25, 37D45, 37A40, 37A05.

Research partially supported by NSF Grant DMS 9801583. 
coefficient is not important to understand dimensionality of $A$, but the exponent is crucial, and it is captured by the following limits:

$$
\lim _{r \rightarrow 0} \frac{\log N(A, r)}{-\log r}=\lim _{r \rightarrow 0} \frac{\log P(A, r)}{-\log r} .
$$

In general these limits fail to exist, and the following two quantities are defined:

$$
\underline{\mathrm{BD}}(A)=\liminf _{r \rightarrow 0} \frac{\log N(A, r)}{-\log r}=\liminf _{r \rightarrow 0} \frac{\log P(A, r)}{-\log r}
$$

and

$$
\overline{\mathrm{BD}}(A)=\limsup _{r \rightarrow 0} \frac{\log N(A, r)}{-\log r}=\limsup _{r \rightarrow 0} \frac{\log P(A, r)}{-\log r} .
$$

The second and forth equality signs in these formulas are due to the fact that minimal covers by balls with radii $r$ and maximal packings with radii $r$ do not differ so much. Namely

$$
P(A, r) \leq N(A, r) \text { and } N(A, 2 r) \leq P(A, r) .
$$

The quantity $\mathrm{BD}(A)$ is called the lower box dimension of $A$ and $\overline{\operatorname{BD}}(A)$ is called the upper box dimension of $A$. If the lower and upper box dimensions coincide, then their common value $\mathrm{BD}(A)=\underline{\mathrm{BD}}(A)=\overline{\mathrm{BD}}(A)$ is called the box dimension of $A$. In the literature all box dimensions are also called, perhaps more properly, box counting dimensions; we will keep here the shorter name. Although the box dimension is a quantity relatively easy to calculate and appreciated by physicists, it behaves rather badly under the simplest topological and set-theoretical operations. For example, $\mathrm{BD}(\bar{A})=\mathrm{BD}(A)$ (so the box dimension of the countable set of all rational numbers from the interval $[0,1]$, is equal to 1 ) and the box dimension of a countable union of sets (even closed) does not have to be equal to the supremum of the box dimensions of its constituents (check that $\operatorname{BD}(\{1 / n: n \geq 1\})=1 / 2$ ). In order to remedy this situation and to capture a finer fractal structure of sets, one considers Hausdorff and packing dimensions. For smooth spaces all these dimensions coincide, but in general they split and can be greater than the topological dimension. In fact, one can reasonably call a set $A$ fractal if at least one of its Hausdorff, packing, or box dimensions is strictly greater than its topological dimension. For our further purposes, let us present now the definition of Hausdorff and packing measures in generalities larger than required just to define Hausdorff and packing dimensions.

Given a nondecreasing function $g:(0, \varepsilon) \rightarrow(0, \infty)$ for some $\varepsilon>0$, the $g$ dimensional outer Hausdorff measure $\mathrm{H}_{g}(A)$ of the set $A$ is defined as

$$
\mathrm{H}_{g}(A)=\sup _{\varepsilon>0} \inf \left\{\sum_{i=1}^{\infty} g\left(\operatorname{diam}\left(A_{i}\right)\right)\right\},
$$

where the infimum is taken over all countable covers $\left\{A_{i}: i \geq 1\right\}$ of $A$ by arbitrary sets whose diameters do not exceed $\varepsilon$. If $g$ is of the form $x^{t}$, instead of writing $\mathrm{H}_{x^{t}}$ we write $\mathrm{H}_{t}$ and speak about $t$-dimensional outer Hausdorff measure. In this case one will get comparable numbers (in the sense that ratios are bounded away from zero and infinity) if instead of covering $A$ by arbitrary sets one considers only open balls centered at points of $A$. 
The $g$-dimensional outer packing measure $\Pi_{g}(A)$ of the set $A$ is defined as

$$
\Pi_{g}(A)=\inf _{\cup A_{i}=A}\left\{\sum_{i} \Pi_{g}^{*}\left(A_{i}\right)\right\}
$$

( $A_{i}$ are arbitrary subsets of $A$ ), where $\Pi_{g}^{*}$, the $g$-packing premeasure, is given by:

$$
\Pi_{g}^{*}(A)=\inf _{\varepsilon>0} \sup \left\{\sum_{i=1}^{\infty} g\left(2 r_{i}\right)\right\} .
$$

Here the supremum is taken over all packings $\left\{B\left(x_{i}, r_{i}\right)\right\}_{i=1}^{\infty}$ of the set $A$ by open balls centered at points of $A$ with radii which do not exceed $\varepsilon$. Similarly as in the case of Hausdorff measures, if $g$ is of the form $x^{t}$, instead of writing $\Pi_{x^{t}}$ we write $\Pi_{t}$ and speak about $t$-dimensional outer packing measure. These two outer measures $\mathrm{H}_{g}$ and $\Pi_{g}$ define countably additive measures on the Borel $\sigma$-algebra of $X$. For additional properties of packing measures and a comprehensive discussion of these and related notions, the reader is referred to the paper [TT] and the books [Mat] and [PU1.

The definitions of the Hausdorff dimension $\operatorname{HD}(A)$ of $A$ and the packing dimension $\mathrm{PD}(A)$ are the following:

$$
\mathrm{HD}(A)=\inf \left\{t: \mathrm{H}_{t}(A)=0\right\}=\sup \left\{t: \mathrm{H}_{t}(A)=\infty\right\}
$$

and

$$
\mathrm{PD}(A)=\inf \left\{t: \Pi_{t}(A)=0\right\}=\sup \left\{t: \Pi_{t}(A)=\infty\right\} .
$$

We recall (see $\mathrm{Fa}$ for example) that $\mathrm{HD}(A) \leq \mathrm{PD}(A) \leq \overline{\mathrm{BD}}(A)$ and $\operatorname{HD}(A) \leq$ $\underline{\mathrm{BD}}(A)=\underline{\mathrm{BD}}(\bar{A}) \leq \overline{\mathrm{BD}}(A)=\overline{\mathrm{BD}}(\bar{A})$. We will discuss in the context of rational functions the cases when these inequalities become equalities.

Let $\nu$ be a Borel probability measure on $X$ and let $t \geq 0$ be a real number. Define the function $\rho=\rho_{t}(\nu): X \times(0, \infty) \rightarrow(0, \infty)$ by

$$
\rho(x, r)=\frac{\nu(B(x, r))}{r^{t}} .
$$

The following two theorems are for our aims the key facts from geometric measure theory. These allow us to determine whether the Hausdorff or packing measure is finite, positive, infinite or whether it vanishes. The proof of the first one follows from the results obtained in $[\mathrm{RT}]$, and the proof of the second one is contained in TT]; cf. also Chapter 6 of the book [PU1].

Theorem 1.1. Assume that $X$ is a compact subset of a d-dimensional Euclidean space. Then for every $t \geq 0$ there exist constants $h_{1}(t)$ and $h_{2}(t)$ with the following properties: If $A$ is a Borel subset of $X$ and $C>0$ is a constant such that

(1) for all (but countably many) $x \in A$

$$
\limsup _{r \rightarrow 0} \rho(x, r) \geq C^{-1},
$$

then for every Borel subset $E \subset A$ we have $\mathrm{H}_{t}(E) \leq h_{1}(t) C \nu(E)$ and, in particular, $\mathrm{H}_{t}(A)<\infty$; or

(2) for all $x \in A$

$$
\limsup _{r \rightarrow 0} \rho(x, r) \leq C^{-1},
$$

then for every Borel subset $E \subset A$ we have $\mathrm{H}_{t}(E) \geq C h_{2}(t) \nu(E)$. 
Theorem 1.2. Assume that $X$ is a compact subspace of a d-dimensional Euclidean space. Then there exist constants $p_{1}(t)$ and $p_{2}(t)$ with the following properties: If $A$ is a Borel subset of $X$ and $C>0$ is a constant such that

(1) for all $x \in A$

$$
\liminf _{r \rightarrow 0} \rho(x, r) \leq C^{-1},
$$

then for every Borel subset $E \subset A$ we have $\Pi_{t}(E) \geq C p_{1}(t) \nu(E)$; or

(2) for all $x \in A$

$$
\liminf _{r \rightarrow 0} \rho(x, r) \geq C^{-1}
$$

then for every Borel subset $E \subset A$ we have $\Pi_{t}(E) \leq p_{2}(t) C \nu(E)$ and, consequently, $\Pi_{t}(A)<\infty$.

$\left(1^{\prime}\right)$ If $\nu$ is non-atomic then (1) holds under the weaker assumption that the hypothesis of part (1) is satisfied on the complement of a countable set.

Frequently a rich fractal structure of a set can be better visualized by examining the structure of its measures. This motivates the following.

Definition 1.3. Given a Borel probability measure $\mu$ on a compact metric space $X$ by $\operatorname{HD}(\mu)$, the Hausdorff dimension of measure $\mu$, we understand the number

$$
\inf \{\operatorname{HD}(Y): \mu(Y)=1\} .
$$

Check, using Theorems 1.1 and 1.2, that the Hausdorff dimension of the Lebesgue measure of a $d$-dimensional unit cube is equal to $d$. Our main tool to understand fractal properties of a Julia set, i.e. its dimensions and measures described above, will be the concept of conformal measures introduced in the case of Fuchsian groups by S. J. Patterson in [Pa1], generalized to the case of Kleinian groups in [Su4] (cf. a thorough presentation given in $\overline{\mathrm{Pa} 2}$ ) and adopted to the case of rational functions by D. Sullivan in [Su1]. The definition of a conformal measure is given below. This is the first and from the fractal point of view the central point of Sullivan's dictionary. We will frequently mention which concepts and theorems have their counterparts in both the theory of iteration of rational functions and the theory of Kleinian groups. This is the level where the analogies are most transparent. They considerably shrink on the level of proofs and almost completely disappear when one deals with the thermodynamic formalism and invariant measures, natural for rational functions and rather artificially adapted in some special cases of Kleinian groups (see [Bo1, $[\mathrm{BS}$, and [KS]).

From now on let $f: \overline{\mathbb{C}} \rightarrow \overline{\mathbb{C}}$ be a rational function of the Riemann sphere $\overline{\mathbb{C}}$. Always assume that the degree of $f$ is at least 2. The main object of our interest will be the Julia set $J(f)$ and the dynamics of $\left.f\right|_{J(f)}$. This dynamics is of chaotic, unpredictable character, whereas the dynamics on its complement $\mathcal{F}$, called the Fatou set of $f$, is stable in the sense of Lyapunov, which means that any two close points stay close forever under positive iterates of $f$. Recall that the Fatou set $\mathcal{F}(f)$ is defined as the set of those points $z \in \overline{\mathbb{C}}$ that admit an open neighbourhood $U$ such that the family of iterates $\left\{\left.f^{n}\right|_{U}\right\}_{n \geq 1}$ is equicontinuous with respect to the spherical metric on $\overline{\mathbb{C}}$. The Julia set $J(f)$ is then defined as $\overline{\mathbb{C}} \backslash \mathcal{F}$. An astonishing result of Montel, which allowed Fatou and Julia to develop an extensive global theory of iterates of rational functions, is that any family of analytic functions which omits three points is equicontinuous. The following basic properties of Julia sets can be found in Bea] or $\mathrm{CG}$. for example. 
(1.a) $J(f)$ is a non-empty compact subset of $\overline{\mathbb{C}}$.

(1.b) $J(f)$ is totally invariant, meaning that $f^{-1}(J(f))=f(J(f))=J(f)$.

(1.c) Either $J(f)=\overline{\mathbb{C}}$ or $J(f)$ is a nowhere dense subset of $\overline{\mathbb{C}}$.

The property (1.b) allows us to consider the dynamical system $f: J(f) \rightarrow J(f)$. The following property completes our list of basic elementary properties of Julia sets $J(f)$.

(1.d) The dynamical system $f: J(f) \rightarrow J(f)$ is topologically exact, i.e. for every non-empty open (in the relative topology) set $U \subset J(f)$ there exists an integer $n \geq 0$ such that $f^{n}(U)=J(f)$.

One can add here that the repelling periodic points are dense in $J(f)$. This property and (1.d) are the first signals of the already mentioned chaotic behavior of $f: J(f) \rightarrow J(f)$.

Definition 1.4. Let $t \geq 0$. A measure $m$ on $J(f)$ is said to be $t$-conformal for $f: \overline{\mathbb{C}} \rightarrow \overline{\mathbb{C}}$ if $m(J(f))=1$ and

$$
m(f(A))=\int_{A}\left|f^{\prime}\right|^{t} d m
$$

for every Borel set $A \subset J(f)$ such that $\left.f\right|_{A}$ is injective. A $t$-conformal measure for some $t \geq 0$ will be called a conformal measure. Since these measures are concentrated on the set $J(f)$, they also will be called conformal for $f: J(f) \rightarrow J(f)$ or conformal for $f$.

In order to motivate this definition notice that if $J(f)=\overline{\mathbb{C}}$ and $t=2$, then the normalized Lebesgue measure is 2-conformal. Even more, if either the $t$-dimensional Hausdorff or packing measure is finite and positive on $J(f)$, then the corresponding normalized Hausdorff or packing measure is $t$-conformal. The issue is, however, to prove that the Hausdorff or packing measure is finite and positive on $J(f)$. So far this is only known in the hyperbolic, parabolic and non-recurrent case, and the main tool to prove this constitutes the concept of conformal measure. Notice also that although conformal, Hausdorff and packing measures share many common features, the first one has a dynamical definition whereas the two others are of a purely geometric character. It is not obvious even in the hyperbolic case that they coincide (they do modulo a constant factor).

In [Su2] Sullivan showed that for every rational mapping $f$ there exists a conformal measure. We briefly sketch his construction below. It parallels the construction performed in the case of Kleinian groups (see [Su4]; cf. [Pa1] and [Pa2]). First, if $J(f)=\overline{\mathbb{C}}$, this measure is the 2-dimensional Lebesgue measure on $\overline{\mathbb{C}}$. Then the exponent will be 2. Otherwise, if $J(f) \neq \overline{\mathbb{C}}$, take a point $z$ in the Fatou set of $f$, the complement of the Julia set, but not in a Siegel disk nor in a Herman ring (the Siegel disks proven to exist by C. L. Siegel in [Si]; cf. [CG. pp. 43 and 86] capture the part of the dynamics which is conjugate to a rotation of the unit disk, while a Herman ring constructed first by M. Herman in 1984 (see [CG, p. 103]) is a structure similar to a Siegel disk, but conjugacy holds only on a topological ring and cannot be extended beyond its boundaries). Such a point must exist since $f^{q}$ restricted to those domains is of degree 1 , where $q$ denotes their period. We may also require $z$ to be the center of a disk $D$ such that $2 D$ is disjoint from

$$
\operatorname{PCV}(f)=\bigcup_{n \geq 1} f^{n}(\operatorname{Crit}(f))=\bigcup_{n \geq 0} f^{n}(f(\operatorname{Crit}(f))),
$$


where

$$
\operatorname{Crit}(f)=\left\{z \in \mathbb{C}: f^{\prime}(z)=0\right\}
$$

is the set of critical points of $f$ and

$$
f(\operatorname{Crit}(f))
$$

is the set of all critical values of $f$. One fairly interesting feature of the set $\mathrm{PCV}(f)$ is that all analytic inverse branches of all forward iterates of $f$ are well defined on simply connected open sets disjoint from $\mathrm{PCV}(f)$. Since for sufficiently small disk $D$ considered above, the sets $f^{-n}(2 D)$ are mutually disjoint (this is why we did not want $z$ to be located in a Siegel disk or a Herman ring), it follows from Koebe's distortion theorem that

$$
\sum_{n \geq 1} \sum_{x \in f^{-n}(z)}\left|\left(f^{n}\right)^{\prime}(x)\right|^{-2}<+\infty .
$$

Now let $\delta \geq 0$ be the infimum of all the exponents $t \geq 0$ for which the series

$$
M_{t}=\sum_{n \geq 1} \sum_{x \in f^{-n}(z)}\left|\left(f^{n}\right)^{\prime}(x)\right|^{-t}
$$

converges. Of course $\delta \leq 2$. For every $s>\delta$ let us consider the following atomic probability measures:

$$
m_{s}=M_{s}^{-1} \sum_{n \geq 1} \sum_{x \in f^{-n}(z)}\left|\left(f^{n}\right)^{\prime}(x)\right|^{-s} \delta_{x}
$$

To conclude the construction of conformal measures, consider any limit point of measures $\left\{m_{s}\right\}$ if $s \searrow \delta$ in the weak topology of probability measures on the sphere $\overline{\mathbb{C}}$. Denote this limit by $m$. This is our candidate for a conformal measure, and we want to discuss the problem of whether $m$ really fulfills the requirements imposed on conformal measures. First, is $m$ concentrated on the Julia set? One can easily notice that if the series $M_{\delta}$ converges, this is not the case. Then simply

$$
m=M_{\delta}^{-1} \sum_{n \geq 1} \sum_{x \in f^{-n}(z)}\left|\left(f^{n}\right)^{\prime}(x)\right|^{-\delta} \delta_{x}
$$

which gives measure 0 to the Julia set. There is, however, a way of overcoming this difficulty by modifying the series $M_{t}$ and the sequence $m_{s}$ a little bit. Namely, one employs so called slowly varying factors. To be more precise, there exists a function $h:(0, \varepsilon) \rightarrow(0,+\infty)$ such that $\lim _{t \rightarrow 0}(h(a t) / h(t))=1$ for every $a>0$ and the series

$$
\sum_{n \geq 1} \sum_{x \in f^{-n}(z)} h\left(\left|\left(f^{n}\right)^{\prime}(x)\right|^{-1}\right)\left|\left(f^{n}\right)^{\prime}(x)\right|^{-\delta}
$$

diverges. Each factor $\left|\left(f^{n}\right)^{\prime}(x)\right|^{-t}$ appearing in the definition of the series $M_{t}$ is then multiplied by $h\left(\left|\left(f^{n}\right)^{\prime}(x)\right|^{-t}\right)$, and each factor $\left|\left(f^{n}\right)^{\prime}(x)\right|^{-s}$ appearing in the definition of the measure $m_{s}$ is multiplied by $h\left(\left|\left(f^{n}\right)^{\prime}(x)\right|^{-s}\right)$. Then, having in mind how the point $z$ was chosen, we conclude that $m(J(f))=1$. It can be verified by an explicit computation that the measure $m$ is conformal.

This construction does not tell us too much about the exponent of conformal measure. In order to get such information, a general scheme of constructing generalizations of conformal measures was proposed in DU1, and it was applied in DU2 to the case of (Sullivan's) conformal measures. This approach is a modification of Patterson's and Sullivan's and the main difference is that we start with 
a point in the Julia set and not in the Fatou set. We will not go into the details here. The interested reader is referred to [DU2] (cf. also [PU1, Chapters 8, 10], and [MM]). Instead, we will describe the precise statements of results from [DU2] and some selected results from Chapters 8-10 of [PU1] that are interesting for us at the moment.

Let us begin with recalling the notion of entropy of a measure-theoretic dynamical system. Suppose that $T: X \rightarrow X$ is a continuous map of a compact metric space $(X, d)$ and that $\mu$ is a Borel $T$-invariant probability measure on $X$. $T$-invariance means that if $A$ is a Borel subset of $X$, then $\mu\left(f^{-1}(A)\right)=\mu(A)$. We call $\mu$ (or $T$ ) ergodic if the only Borel invariant subsets of $T$ (i.e. satisfying $T^{-1}(A)=A$ ) are either of measure 0 or 1 . Given $n \geq 0$ we define the metric $d_{n}$ on $X$ by setting

$$
d_{n}(x, y)=\max \left\{d\left(T^{i}(x), T^{i}(y)\right): 0 \leq i \leq n\right\} .
$$

Denote by $B_{n}(x, r)$ the open ball in the metric $d_{n}$ centered in $x$ and with radius $r$. If the measure $\mu$ is ergodic, then (see $[\mathrm{BK}]$ ) for $\mu$-a.e. point $x \in X$ the limit

$$
\lim _{r \rightarrow 0} \lim _{n \rightarrow \infty}-\frac{\log \mu\left(B_{n}(x, r)\right)}{n}
$$

exists, is independent of $x$, and is called the (metric) entropy of the system $T$ with respect to the measure $\mu$. This entropy is denoted by $\mathrm{h}_{\mu}(T)$. Roughly speaking it measures the exponential rate of decay of the measure of points that stay $\varepsilon$-close to the point $x$ under forward iterates of $f$. Observe that the entropy of any isometry with respect to any invariant measure is always equal to zero, whereas the entropy of the map $z \mapsto z^{d}, z \in S^{1}=\{z \in \mathbb{C}:|z|=1\}$, with respect to the normalized arc-length measure on $S^{1}$, is equal to $\log d$. Usually a different, more classical, approach is undertaken to define the entropy $\mathrm{h}_{\mu}(T)$ (see [BK], Wa1], and [HK] for example); the one chosen here is probably the fastest and, at the same time, reflects in a better way the nature of entropy.

If $\mu$ is a Borel probability measure on the Julia set of a rational function $f: \overline{\mathbb{C}} \rightarrow$ $\overline{\mathbb{C}}$, then $\int \log \left|f^{\prime}\right| d \mu \leq \log \left\|f^{\prime}\right\|<\infty$ (we mean here the derivative calculated with respect to the spherical metric and $\|\cdot\|$ the supremum metric over $\overline{\mathbb{C}}$ ) and, as was proved in [Pr1], $\int \log \left|f^{\prime}\right| d \mu \geq 0$. We call the integral $\int \log \left|f^{\prime}\right| d \mu$ the Lyapunov (characteristic) exponent of $f$ with respect to the measure $\mu$ and denote it by $\chi_{\mu}(f)$. The following theorem was proved in [Ma1] (cf. also [Pr2 and Chapters 810 of [PU1), and we include its proof in the hyperbolic case (next section) for Gibbs measures.

Theorem 1.5. If $f: \overline{\mathbb{C}} \rightarrow \overline{\mathbb{C}}$ is a rational function and $\mu$ is a Borel probability ergodic invariant measure on $\overline{\mathbb{C}}$ such that $\chi_{\mu}(f)>0$, then

$$
\operatorname{HD}(\mu)=\frac{h_{\mu}(f)}{\chi_{\mu}(f)} .
$$

We would like to point out that the ergodicity of $f$ and the fact that the Lyapunov exponent is positive imply that $\mu$ is supported on the Julia set. We would also like to add that due to Ruelle's inequality, $\mathrm{h}_{\mu}(f) \leq 2 \chi_{\mu}(f)$, the inequality $\chi_{\mu}(f)>0$ is implied by the inequality $\mathrm{h}_{\mu}(f)>0$. See that due to Theorem 1.5 the purely geometric quantity $\operatorname{HD}(\mu)$ has been expressed by purely dynamical terms $\mathrm{h}_{\mu}(f)$ and $\chi_{\mu}(f)$. This makes it conceivable that the Hausdorff dimension of the entire Julia set can always be expressed in dynamical terms. Following this path, it is natural to introduce the following definition coming from DU2]. 
Definition 1.6. The dynamical dimension $\mathrm{DD}(J(f))$ is defined as

$$
\mathrm{DD}(J(f))=\sup \{\mathrm{HD}(\mu)\},
$$

where the supremum is taken over all ergodic invariant measures of positive entropy.

We call a compact forward invariant subset $X \subset J(f)$ (i.e. satisfying property $f(X) \subset X)$ hyperbolic if there exists $n \geq 1$ such that

$$
\left|\left(f^{n}\right)^{\prime}(x)\right|>1
$$

for every $x \in X$ and $f^{n}$ is topologically conjugate to a subshift of finite type. If only condition $\left|\left(f^{n}\right)^{\prime}(x)\right|>1$ is satisfied, we call the map $\left.f\right|_{X}$ expanding. Since the dynamics and ergodic theory of subshifts of finite type are fairly well understood, it would be nice to know that the hyperbolic sets approximate in some sense the entire set $J(f)$, for example that their Hausdorff dimensions approximate the Hausdorff dimension of $J(f)$. This justifies the following definition (see [Sh]; cf. [PU1]).

Definition 1.7. The hyperbolic dimension $\mathrm{hD}(J(f))$ is defined to be the supremum of Hausdorff dimensions of all hyperbolic subsets of $J(f)$.

The next definition aims to select, in a certain sense, the best of all conformal measures.

Definition 1.8. $\delta(f)$ is the minimal exponent for which a conformal measure exists.

We are now in the position to formulate the main result of this section.

Theorem 1.9. For all rational functions $f: \overline{\mathbb{C}} \rightarrow \overline{\mathbb{C}}$ we have that

$$
\mathrm{DD}(J(f))=\mathrm{hD}(J(f))=\delta(f) .
$$

The equality of the numbers $\mathrm{DD}(J(f))$ and $\delta(f)$ was essentially proved in DU2. The proof became complete after F. Przytycki demonstrated in Pr1] that each compact forward invariant subset of $J(f)$ contains points $z$ for which $\limsup _{n \rightarrow \infty}\left|\left(f^{n}\right)^{\prime}(z)\right|=\infty$. The reader may also consult Mc1 for the proof. The equality $\mathrm{DD}(J(f))=\mathrm{hD}(J(f))$ has been shown in Chapter 8 of [PU1]. The general scheme of the proof of Theorem 1.9 is the following: First, it follows easily from the classical Bowen type formula (see [Bo1]; cf. Chapter 6 of [PU1]) that $\mathrm{hD}(J(f)) \leq \mathrm{DD}(J(f))$. A generalization of Bowen's formula will be discussed in Sections 2 and 3, which are devoted to hyperbolic and parabolic rational functions. Second, using an appropriate version of Pesin's theory and Katok's type considerations (see Chapter 8 of [PU1]), one shows that $\mathrm{hD}(J(f)) \geq \mathrm{DD}(J(f))$. The easiest part (modulo Pesin's theory and Katok-Strelcyn type results (see Chapter 8 of (PU1)) is to show that $\mathrm{hD}(J(f)) \leq \delta(f)$. One sees here the fact that each ergodic invariant measure of positive entropy gives rise to a subset of the set of conical points of full measure. The set of conical points will be discussed in greater detail soon. At this moment let us only informally say that conical points are those whose forward iterates allow infinitely often univalent analytic pull-backs from some fixed scale. Finally, developing the technique of so called compact invariant $K(V)$-sets and defining on them classical topological pressure of the functions $-t \log \left|f^{\prime}\right|, t \in \mathbb{R}$, one constructs a conformal measure with exponent $\leq \mathrm{DD}(J(f))$. This proves the inequality $\delta(f) \leq \mathrm{DD}(J(f))$ and finishes the proof of Theorem 1.9.

A natural byproduct of the proof of Theorem 1.9 is the appearance of the concept of conical points. They correspond to conical points in the theory of Kleinian groups and were introduced in U1] (cf. also [DMNU] and [Mc1]). Here is their definition. 
Definition 1.10. A point $z \in J(f)$ is called conical for $f$ if there exist $\theta>0$ and an infinite increasing sequence $n_{k} \geq 1$ of positive integers such that for each $k$ there exists $f_{z}^{-n_{k}}$, a holomorphic inverse branch of $f^{n_{k}}$, which is defined on the disk $B\left(f^{n_{k}}(z), \theta\right)$ and sends the point $f^{n_{k}}(z)$ to $z$. The set of all conical points of $f$ will be denoted by $J_{c}(f)$. A conical point is called uniformly conical if one can find a sequence $\left\{n_{k}\right\}$ such that

$$
\limsup _{n \rightarrow \infty} \frac{\left|\left(f^{n_{k+1}}\right)^{\prime}(z)\right|}{\left|\left(f^{n_{k}}\right)^{\prime}(z)\right|}<\infty .
$$

Using Koebe's distortion theorem one can easily see that for every conical point $z$ there exists a sequence of radii $\left\{r_{k}(z)\right\}_{k=1}^{\infty}$ converging to $0\left(r_{k}(z)\right.$ is approximately equal to $\left.\theta\left|\left(f^{n_{k}}\right)^{\prime}(z)\right|^{-1}\right)$ such that if $m$ is a $t$-conformal measure on $J(f)$, then

$$
C^{-1} \leq \frac{m\left(B\left(z, r_{k}(z)\right)\right)}{r_{k}^{t}(z)} \leq C
$$

for some constant $C$ depending on $\theta$ and $m$. The reader may acknowledge the importance of this formula by looking back at Theorem 1.1 and Theorem 1.2. In DMNU] we have proved the following.

Theorem 1.11. There exists at most one value of $t$ for which a t-conformal measure exists and is supported on the set of conical points of $f$. Additionally, for such a t there is exactly one $t$-conformal measure supported on the set of conical points of $f$.

We would like to end up the discussion concentrated around Theorem 1.9 with two problems.

Problem 1.12. Is the Hausdorff dimension of the Julia set always equal to its dynamical dimension?

Problem 1.13. Does there always exist a conformal measure supported on the set of conical points?

Some partial positive answers to these problems will be given in the following sections. In general, the answer to Problem 1.13 is negative. Indeed, it has been recently communicated to me by Volker Mayer May that the quadratic polynomials constructed by Shishikura (see [Sh]) having dynamical dimension equal to 2 and vanishing 2-dimensional Lebesgue measure admit no conformal measures supported on conical points. Indeed, suppose on the contrary that such a polynomial has a $t$-conformal measure supported on conical points. Since the dynamical dimension is equal to $2, t=2$. It then easily follows from (1.2) that the Lebesgue measure would have to be positive.

We shall end this section with an estimate from below of the Hausdorff dimension of the Julia set which is caused by the existence of so called parabolic points. First we need the following.

Definition 1.14. A periodic point $z$ of $f$, say of period $q \geq 1$, is called parabolic if the derivative $\left(f^{q}\right)^{\prime}(z)$ is a root of unity.

In the literature parabolic points are frequently called rationally indifferent or neutral periodic points. A Fatou theorem says (see [Bea] and [CG] for example) that the set $\Omega$ of all parabolic points is finite and contained in the Julia set. Suppose now that $\omega \in \Omega$ is a fixed point of $f$. Looking at the Taylor series expansion of 
$f$ around $\omega$ and at Fatou's flower theorem (see [Bea] and [CG] for example), one can deduce (see [ADU]) that there exists $\sigma>0$ such that for every sufficiently small $\delta>0$ and every point $z \in B(\omega, \delta) \backslash\{\omega\}$ all continuous inverse branches $f_{\omega}^{-n}: B(z, \sigma|z-\omega|) \rightarrow \mathbb{C}, n \geq 1$, of $f^{n}$ sending point $z$ closer to $\omega$ are well defined. Looking again at the Taylor series expansion of $f$ around $\omega$, one can conclude (see [ADU]) that for every $x \in B(z, \sigma|z-\omega|)$

$$
\left|\left(f_{\omega}^{-n}\right)^{\prime}(x)\right| \asymp n^{-(p+1) / p}
$$

and

$$
\left|f_{\omega}^{-n}(x)-\omega\right| \asymp n^{-1 / p}
$$

where $p=p(\omega)$ is the number of petals associated with the parabolic point $\omega$ and where the comparability constants depend only on the distance of $z$ to $\omega$. Relying on (1.3) and following the proof of Theorem 8.5 in [ADU] (see also [Mc1]), we shall provide a simple argument for the following.

Theorem 1.15. If a rational function $f: \overline{\mathbb{C}} \rightarrow \overline{\mathbb{C}}$ has a parabolic point with $p$ petals, then $\operatorname{HD}(J(f))>p /(p+1)$.

Proof. Let $\omega$ be a parabolic point of $f$. Passing to a sufficiently high iterate we may assume that $f$ fixes $\omega$. Consider now a point $z \in J(f) \cap(B(\omega, \delta) \backslash\{\omega\})$ and let $V \subset B(z, \sigma|z-\omega|)$ be a neighbourhood of $z$ which is chosen so small that all the inverse images $f_{\omega}^{-n}(V), n \geq 0$, are mutually disjoint. Let $m$ be an arbitrary conformal measure for $f: J(f) \rightarrow J(f)$ and let $h=\operatorname{HD}(J(f))$. Since $z \in J(f)$, $m(V)>0$. Using (1.3) and Koebe's distortion theorem we obtain the following:

$$
1 \geq m\left(\bigcup_{n \geq 1} f_{\omega}^{-n}(V)\right)=\sum_{n \geq 1} m\left(f_{\omega}^{-n}(V)\right) \asymp \sum_{n \geq 1} n \frac{\frac{-(p+1) h}{p}}{p} m(V) .
$$

Thus $h>p /(p+1)$ and the proof is complete.

We would like to conclude this section with the remark that McMullen's paper Mc3 contains an algorithm for computing the Hausdorff dimension of any conformal hyperbolic set up to any desired accuracy. Also, in this paper lots of various examples are treated.

\section{Hyperbolic RATiOnAL FUnCTIONS}

Hyperbolic rational maps form the most thoroughly understood subclass of rational functions. They serve as a model for investigations of other subclasses of rational functions, as for example parabolic maps, NCP maps or Collet-Eckmann maps (see following sections). The geometric structure of hyperbolic Julia sets exhibits the same formal properties as the structure of the limit sets of convex co-compact Kleinian groups. In particular Theorems 1.1 and 1.2 are true in exactly the same form for these groups. This is a clear, transparent part of Sullivan's dictionary. However the analogy breaks down when one passes to the concepts of topological pressure and invariant measures. These have some counterparts in the theory of Kleinian groups in some particular cases (see [Bo1], [BS], and the recent article [KS] by M. Kesseböhme and B. Stratmann) and are by no means canonical.

In this survey we will present only basic geometric facts about hyperbolic maps. This will equip the reader with the language and techniques sufficient to continue investigations of this particular class as well as to deal with larger classes of rational 
functions. At the end of this section we only briefly touch on four out of potentially infinitely many possible continuations of the research on hyperbolic rational functions. One of the most systematic treatments of hyperbolic Julia sets which form the most transparent class of conformal expanding repellers can be found in Chapter 7 of the book [PU1].

The central point of our exposition in this section (and others as well) is the concept of conformal measures introduced in the previous section. In particular, in order to study the geometry of Julia sets we do not need to use Markov partitions. This is of some importance since the NCP maps treated in Section 4 do not have Markov partitions and, although parabolic maps admit them, their diameters under backward iterates do not converge to zero exponentially fast and not all of them have bounded distortion.

Definition 2.1. A rational function $f: J(f) \rightarrow J(f)$ is called hyperbolic if there exists $n \geq 1$ such that

$$
\inf \left\{\left|\left(f^{n}\right)^{\prime}(z)\right|: z \in J(f)\right\}>1 .
$$

This definition coincides with the definition of a hyperbolic set given right after Definition 1.6, since each hyperbolic rational function is topologically conjugate to a subshift of finite type. Let us now look closer at the polynomials $z \mapsto z^{n}$, $n \geq 2$. The points inside the open unit disk converge locally uniformly to zero under forward iterates of these polynomials, the points outside the closure of the unit disk converge locally uniformly to infinity, whereas the points on the unit circle $S^{1}=\{z \in \mathbb{C}:|z|=1\}$ stay on $S^{1}$ forever. Consequently, the Julia set of any map $z \mapsto z^{n}, n \geq 2$, is equal to the unit circle $S^{1}$. Since $\left|\left(z^{n}\right)^{\prime}\right|=n \geq 2$ on $S^{1}$, we see that each map $z \mapsto z^{n}, n \geq 2$, is hyperbolic. Recall that $\operatorname{PCV}(f)$ is the forward trajectory of the set of critical values of $f$. The following topological characterization of hyperbolicity, usually much more suitable for verification than the original definition, was known already to Fatou and Julia (see Bea and [CG] for example and the references therein).

Theorem 2.2. A rational function $f: J(f) \rightarrow J(f)$ is hyperbolic if and only if

$$
\overline{\mathrm{PCV}(f)} \cap J(f)=\emptyset \text {. }
$$

It immediately follows from this theorem that each point of a hyperbolic Julia set is conical (see Definition 1.10), in fact is uniformly conical. The same statement is true for convex co-compact Kleinian groups - one item more to Sullivan's dictionary. This gives an immediate positive answer to Problem 1.13 in the class of hyperbolic rational functions.

Imagine now that you perturb the map $z \mapsto z^{2}$ a little bit, say to a map $f_{\varepsilon}(z)=$ $z^{2}+\varepsilon$, where $\varepsilon$ is very close to zero. Then the disk $B(0,1 / 2)$ is compactly mapped into itself. Thus due to Montel's theorem, the family $f_{\varepsilon}^{n}: B(0,1 / 2) \rightarrow B(0,1 / 2)$ is equicontinuous. Hence, the ball $B(0,1 / 2)$ is contained in the Fatou set of $f_{\varepsilon}$. Since

$$
\operatorname{PCV}(f)=\left\{f_{\varepsilon}^{n}(0): n \geq 1\right\} \cup\{\infty\} \subset B(0,1 / 2) \cup\{\infty\} \subset \mathcal{F}(f),
$$

Theorem 2.2 implies that all the maps $f_{\varepsilon},|\varepsilon|<<1$, are hyperbolic.

Now look again at the simplest example of a hyperbolic map $f(z)=z^{2}$. Then the normalized arc-length measure $m_{1}$ on $S^{1}$ is 1-conformal for $f$, the $m_{1}$ measure of any sufficiently small ball centered at a point in $S^{1}$ is comparable with the length of its radius, the Hausdorff dimension of the Julia set $S^{1}$ is equal to 1, and all three 
measures - conformal $m_{1}$, Hausdorff $\mathrm{H}_{1}$ and packing $\Pi_{1}$ - coincide on $S^{1}$ up to multiplicative constants. As the next theorem shows, these nice fractal properties, appropriately modified, continue to be true for all hyperbolic maps.

Theorem 2.3. If $m_{t}$ is a $t$-conformal measure for a hyperbolic rational function $f: \overline{\mathbb{C}} \rightarrow \overline{\mathbb{C}}$, then

(a) There exists $C \geq 1$ such that for all $r \in(0,1]$

$$
C^{-1} \leq \frac{m_{t}(B(x, r))}{r^{t}} \leq C .
$$

(b) $t=\mathrm{HD}(J(f))$.

(c) The measures $m_{t},\left.\mathrm{H}_{t}\right|_{J(f)}$ and $\left.\Pi_{t}\right|_{J(f)}$ coincide up to a multiplicative constant.

Proof. In this proof we will use Koebe's distortion theorem, although we would like to emphasize that in the case of hyperbolic rational functions this theorem can be proved by purely dynamical means as a special case of bounded distortion property valid in the setting of distance expanding continuous maps and Hölder continuous potentials (see for example [PU1).

(a) Given $0<r \leq 1$ and $x \in J(f)$ fix $n \geq 1$ determined by the condition that $r\left|\left(f^{n}\right)^{\prime}(x)\right| \xi \asymp 1$, where $\xi=\frac{1}{2} \operatorname{dist}(J(f), \overline{\mathrm{PCV}})>0$. It then follows from Koebe's distortion theorem that $B(x, r) \asymp f_{x}^{-n}\left(B\left(f^{n}(x), \xi\right)\right)$, where $f_{x}^{-n}: B\left(f^{n}(x), \xi\right) \rightarrow \mathbb{C}$ is the holomorphic inverse branch of $f^{n}$ sending $f^{n}(x)$ to $x$, and that

$$
\begin{aligned}
m_{t}(B(x, r)) & =\int_{B\left(f^{n}(x), \xi\right)}\left|\left(f_{x}^{-n}\right)^{\prime}(z)\right|^{t} d m_{t}(z) \asymp\left|\left(f^{n}\right)^{\prime}(x)\right|^{-t} m_{t}\left(B\left(f^{n}(x), \xi\right)\right) \\
& \asymp\left|\left(f^{n}\right)^{\prime}(x)\right|^{-t} \asymp r^{t} .
\end{aligned}
$$

The proof of part (a) is complete.

(b) This is an immediate consequence of (a) and Theorem 1.1.

(c) It immediately follows from (a) along with Theorems 1.1 and 1.2 that all three measures appearing in item (c) are mutually equivalent with Radon-Nikodym derivatives bounded away from zero and infinity. Since all these Radon-Nikodym derivatives are constant along orbits of $f$, ergodicity of measure $m_{t}$ (see the discussion of equilibrium states and Gibbs states of Hölder continuous potentials below) completes the proof.

Notice that it follows from items (b) and (c) of Theorem 2.3 that there exists exactly one (in contrast with parabolic and non-recurrent cases) exponent $t$ for which a $t$-conformal measure exists, namely $t=\operatorname{HD}(J(f))$. In addition, there exists exactly one $\operatorname{HD}(J(f))$-conformal measure. As an immediate consequence of Theorem 2.3(a) and (b), we get the following.

Theorem 2.4. If $f: \overline{\mathbb{C}} \rightarrow \overline{\mathbb{C}}$ is a hyperbolic rational function, then $\overline{\operatorname{BD}}(J(F))=$ $\mathrm{HD}(J(f))$.

We would like to notice that this simple fact in the theory of hyperbolic rational functions has a much more involved proof in the parabolic case (see [DU5]) and is not established for all NCP maps (see Section 4).

An intriguing open problem is whether there exist nowhere dense Julia sets whose Lebesgue measure is positive. In the class of hyperbolic maps such examples do not exist. As the following theorem shows, even more is true. 
Theorem 2.5. If $f: \overline{\mathbb{C}} \rightarrow \overline{\mathbb{C}}$ is a hyperbolic rational function, then $\overline{\operatorname{BD}}(J(F))=$ $\operatorname{HD}(J(f))<2$.

Proof. (Sketch) First notice that since all points of the Julia set of $f$ are conical, one drags from the large scale holes in the Julia set to arbitrarily small neighborhoods of all points of $J(f)$ and therefore, due to Koebe's Distortion Theorem, for every $x \in J(f)$ we have that

$$
\liminf _{r \rightarrow 0} \frac{\lambda(J(f) \cap B(x, r))}{\lambda(B(x, r))}<1 .
$$

This, due to Lebesgue's Density Theorem, implies that $\lambda(J(f))=0$. Suppose on the contrary that $h=\operatorname{HD}(J(f))=2$. Then Theorem 2.3(a) would imply that $m_{2}$ and the Lebesgue measure on $J(f)$ are equivalent. In particular $\lambda(J(f))>0$. This contradiction finishes the proof.

We will add more details to this argument in the last part of the proof of Theorem 3.8 in context of parabolic rational functions. We would like to mention here that Theorem 2.5 follows immediately from porosity (see the paragraph preceding Theorem 3.9) of hyperbolic Julia sets and Theorem 3.9. The porosity of hyperbolic Julia sets is trivial: as in the first part of the proof of Theorem 2.5 one drags holes in the Julia set from the large scale to a neighbourhood of a given point $x \in J(f)$, but one needs to notice in addition that they will be contained in geometric annuli centered at $x$ with radii decreasing to zero as a geometric sequence.

In order to obtain a more dynamical description of Hausdorff and packing measures and a dynamical interpretation of the Hausdorff dimension, we need first the concept of topological pressure (see [Ru, Wa1, Wa2, Bo2] and [PU1 for example). In order to introduce it, we choose one of the fastest methods. For alternative approaches better suited to derive various properties of topological pressure, see also the positions quoted above. Consider a continuous mapping $T: X \rightarrow X$ of a compact metric space $(X, d)$ and a continuous function $\phi: X \rightarrow \mathbb{R}$ called, following physical tradition, a potential. Given $n \geq 0$ and $\varepsilon>0$, we say that a subset $F$ of $X$ is $(n, \varepsilon)$-separated if it is $\varepsilon$-separated with respect to the metric $d_{n}$, which means that if $x$ and $y$ are two distinct points of $F$, then $d_{n}(x, y) \geq \varepsilon$. Fixing $\varepsilon>0$ we consider an arbitrary sequence $F_{n}(\varepsilon), n \geq 1$, of maximal (in the sense of inclusion) $(n, \varepsilon)$-separated sets. We then define the topological pressure of the function $\phi$ with respect to the mapping $T$ as follows:

$$
\mathrm{P}(T, \phi)=\lim _{\varepsilon \rightarrow 0} \limsup _{n \rightarrow \infty} \frac{1}{n} \log \left(\sum_{x \in F_{n}(\varepsilon)} \exp \sum_{j=0}^{n-1} \phi \circ T^{j}(x)\right) .
$$

Observe that in the case of expansive maps (see the beginning of Section 3 for the appropriate definition) it is not necessary to take the limit for $\varepsilon \rightarrow 0$; it is then enough to take $\varepsilon$ as an expansive constant of $T$. In the case when $\phi=0$, the topological pressure is called topological entropy. It has been proved (see $[\mathrm{Gr}]$ and Ly2 ) that topological entropy of any rational function is equal to the logarithm of its degree. Topological pressure belongs to topological dynamics, whereas metric entropy is a notion in ergodic theory. The link joining them is given by the following formula called the variational principle (see [Ru], Wa1], Wa2], [Bo2] and [PU1] for 
example):

$$
\mathrm{P}(T, \phi)=\sup _{\mu}\left\{\mathrm{h}_{\mu}(T)+\int \phi d \mu\right\},
$$

where the supremum is taken over all Borel probability $T$-invariant (ergodic) measures of $X$.

In the case when $T: X \rightarrow X$ is an open distance expanding map (the latter means that there are constants $\zeta>0$ and $\lambda>1$ such that if $d(x, y)<\zeta$, then $d(T(x), T(y)) \geq \lambda d(x, y))$ and $\phi: X \rightarrow \mathbb{R}$ is a Hölder continuous function, there exists a unique $T$-invariant measure $\mu_{\phi}$, called the equilibrium state of the potential $\phi$, such that $\mathrm{h}_{\mu_{\phi}}(T)+\int \phi d \mu_{\phi}=\mathrm{P}(T, \phi)$. In particular $\mu_{\phi}$ is ergodic. Among all the Borel probability $T$-invariant measures, $\mu_{\phi}$ is characterized by the condition that for every $r>0$ small enough there exists a constant $C_{r} \geq 1$ such that

$$
C_{r}^{-1} \leq \frac{\mu_{\phi}\left(B_{n}(x, r)\right)}{\exp \left(S_{n} \phi(x)-\mathrm{P}(\phi) n\right)} \leq C_{r}
$$

for every $x \in X$ and all $n \geq 1$. Here

$$
S_{n} \phi=\sum_{j=0}^{n-1} \phi \circ T^{j} .
$$

Because of the property (2.1) the equilibrium state $\mu_{\phi}$ is frequently called the Gibbs state of the potential $\phi$. For a systematic exposition of the theory of Gibbs states for the distance expanding maps, the reader may consult Chapter 3 of the book PU1] as well as [Bo2] and [Ru]. We may assume without loss of generality that $\mathrm{P}(\phi)=0$. In the case of hyperbolic Julia sets condition (2.1) easily translates into the following. There exists $E \geq 1$ such that for all $x \in J(f)$ and all $r>0$ small enough

$$
E^{-1} \leq \frac{\mu_{\phi}(B(x, r))}{\exp S_{n} \phi(x)} \leq E,
$$

where $n=n(x, r)$ is the largest integer such that $\left|\left(f^{n}\right)^{\prime}(x)\right| r C \leq \xi$, and let us recall $\xi=\frac{1}{2} \operatorname{dist}(J(f), \overline{\mathrm{PCV}})$. With its help it is possible to provide a complete proof of Theorem 1.5 assuming that $\mu$ is a Gibbs state. In the general case the idea of the proof is the same, and instead of (2.2) one uses the Brin-Katok (see the second to last paragraph above Theorem 1.5) definition of metric entropy.

Proof of Theorem 1.7 in the case when $\mu=\mu_{\phi}$ is the Gibbs state of a Hölder continuous potential. First notice that in view of Theorem 1.3 it suffices to show that for $\mu_{\phi}$-almost every point $x \in J(f)$

$$
\lim _{r \rightarrow 0} \frac{\log \mu_{\phi}(B(x, r))}{\log r}=\frac{\mathrm{h}_{\mu_{\phi}}(f)}{\chi_{\mu_{\phi}}(f)} .
$$

In order to prove this formula notice first that for $n=n(x, r)$ we have

$$
-\log E+S_{n} \phi(x) \leq \log \mu_{\phi}(B(x, r)) \leq \log E+S_{n} \phi(x)
$$

and

$$
\log \left(\xi\left(C|| f^{\prime}||\right)^{-1}\right)-\log \left|\left(f^{n}\right)^{\prime}(x)\right| \leq \log r \leq \log (\xi C)-\log \left|\left(f^{n}\right)^{\prime}(x)\right| .
$$


Combining these four inequalities we obtain

$$
\frac{-\log E+S_{n} \phi(x)}{\log \left(\xi C^{-1}|| f^{\prime}||^{-1}\right)-\log \left|\left(f^{n}\right)^{\prime}(x)\right|} \leq \frac{\log \left(\mu_{\phi}(B(x, r))\right.}{\log r} \leq \frac{\log E+S_{n} \phi(x)}{\log (\xi C)-\log \left|\left(f^{n}\right)^{\prime}(x)\right|}
$$

Now, in view of Birkhoff's Ergodic Theorem, there exists a Borel set $X \subset J(f)$ such that $\mu_{\phi}(X)=1$ and for all $x \in X$

$$
\lim _{n \rightarrow \infty} \frac{1}{n} S_{n} \phi(x)=\int \phi d \mu_{\phi} \text { and } \lim _{n \rightarrow \infty} \frac{1}{n} \log \left|\left(f^{n}\right)^{\prime}(x)\right|=\chi_{\mu_{\phi}}(f) .
$$

Keeping in mind that $\mathrm{h}_{\mu_{\phi}}(f)+\int \phi d \mu_{\phi}=\mathrm{P}(\phi)=0$ and combining the last four inequalities along with the observation that $n \rightarrow \infty$ as $r \rightarrow 0$, we conclude that for all $x \in X$

$$
\lim _{r \rightarrow 0} \frac{\log \mu_{\phi}(B(x, r))}{\log r}=\frac{\int \phi d \mu_{\phi}}{-\chi_{\mu_{\phi}}(f)}=\frac{\mathrm{h}_{\mu_{\phi}}(f)}{\chi_{\mu_{\phi}}(f)} .
$$

The proof is finished.

Since the Julia set of a hyperbolic rational function $f: J(f) \rightarrow J(f)$ contains no critical points, we may define the function

$$
\mathrm{P}(t)=\mathrm{P}\left(f,-t \log \left|f^{\prime}\right|\right), t \in \mathbb{R}
$$

which is called the pressure function. Since $n \mathrm{P}(t)=\mathrm{P}\left(f^{n},-t \log \left|\left(f^{n}\right)^{\prime}\right|\right)$, hyperbolicity of $f$ implies that the function $t \mapsto \mathrm{P}(t)$ is strictly decreasing, $\lim _{t \rightarrow-\infty} \mathrm{P}(t)=$ $+\infty$ and $\lim _{t \rightarrow+\infty} \mathrm{P}(t)=-\infty$. In addition it is easy to see that the function $\mathrm{P}(t)$ is convex and consequently continuous. In fact it is real-analytic. We are now in position to sketch the proof of the following theorem (see [Bo1]; cf. [MM] and PU1]) called the Bowen-Manning-McCluskey formula.

Theorem 2.6. If $f: \overline{\mathbb{C}} \rightarrow \overline{\mathbb{C}}$ is a hyperbolic rational function, then $h=\operatorname{HD}(J(f))$ is the only zero of the pressure function $t \mapsto \mathrm{P}(t), t \in \mathbb{R}$. In addition, the (ergodic) Gibbs state $\mu_{h}=\mu_{-h \log \left|f^{\prime}\right|}$ is equivalent to the conformal measure $m_{h}$ with RadonNikodem derivatives bounded away from zero and infinity.

Proof. Let $h$ be the only zero of $\mathrm{P}(t)$. In view of (2.2) for every $r>0$ small enough and every $x \in J(f)$ we have

$$
\frac{\mu_{h}(B(x, r))}{\left|\left(f^{n(x, r)}\right)^{\prime}(x)\right|^{-h}} \asymp 1 .
$$

But since $\left|\left(f^{n(x, r)}\right)^{\prime}(x)\right| \asymp r^{-1}$, we get

$$
\frac{\mu_{h}(B(x, r))}{r^{h}} \asymp 1 \text {. }
$$

This formula along with Theorem 2.3 and Theorem 1.1 finishes the proof.

Since each Gibbs state of a Hölder continuous potential has positive entropy, it immediately follows from this definition that for hyperbolic rational functions the answer to Problem 1.12 is positive.

The theory of hyperbolic rational functions (not to mention its generalizations) has developed in numerous still active directions. Let me only point out its connections with the potential theory, especially with its part devoted to the study of geometric properties of harmonic measure in the simply connected case (see Man, Pr2, PUZ, I], $\mid \overline{P U Z}, \mathrm{II}$ for example) as well as in the totally disconnected case (see [Ca], Vo], UZ and references therein). 
Another interesting subfield of hyperbolic systems concerns multifractal analysis of Gibbs states of Hölder continuous potentials. This topic, which has a long history, has been rigorously and thoroughly investigated by Ya. Pesin and H. Weiss (see [Pe], [PW]; cf. Chapter 7 of [PU1]).

We would also like to add that there exists a huge theory of stochastic and statistical properties of Gibbs states. Its results rely mostly on the possibility of constructing Markov partitions and working with the corresponding symbolic representation of hyperbolic systems. As an introduction to this theory the reader may consult the first and third chapters of the book [PU1]. A large part of investigations of harmonic measure in the context of hyperbolic maps depends heavily on stochastic properties (Central Limit Theorem, the Law of Iterated Logarithm) of Gibbs states.

Let us mention finally the rigidity theory originated by D. Sullivan in Su3]. It goes far beyond the hyperbolic case, as the reader may see in Appendix 1.

\section{Parabolic Rational Functions}

We call a continuous map $T: X \rightarrow X$ from a compact metric space $(X, d)$ into itself expansive if there exists $\delta>0$ such that for all pairs of points $x, y \in X, x \neq y$, there exists $n \geq 0$ such that $d_{n}(x, y) \geq \delta$. Any number $\delta$ satisfying this property will be called an expansive constant. In [DU3] we have proved the following topological characterization of expansive rational functions $f: J(f) \rightarrow J(f)$ in the spirit of the topological characterization of hyperbolic rational functions given in Theorem 2.2.

Theorem 3.1. A rational function $f: J(f) \rightarrow J(f)$ is expansive if and only if the Julia set $J(f)$ contains no critical points of $f$.

One direction of this equivalence is simple. Namely, if the Julia set $J(f)$ contains a critical point, say $c$, then $f: J(f) \rightarrow J(f)$ is not expansive. To see this, notice that by perfectness of $J(f)$ we can find a sequence $x_{n}, n \geq 1$, of points in $J(f)$ converging to $c$. Since $c$ is a critical point of $f$, for each point $x_{n}$ (sufficiently close to $c$ ) there exists a different point $y_{n} \neq x_{n}$ such that

$$
f\left(y_{n}\right)=f\left(x_{n}\right) .
$$

By (1.b) $y_{n} \in J(f)$. In addition, we may require that $\lim _{n \rightarrow \infty} y_{n}=c$ and, in particular, that $\lim _{n \rightarrow \infty}\left|y_{n}-x_{n}\right|=0$. This together with (3.1) contradicts expansiveness. The other direction is more subtle and requires good control of the inverse branches.

It follows from Theorems 3.1 and 2.2 that each hyperbolic rational function is expansive and that a rational function is expansive but not hyperbolic if and only if the Julia set contains no critical points of $f$ but intersects the $\omega$-limit set of critical points. Now, if we have a rational function whose Julia set $J(f)$ contains no critical points of $f$, then it follows from the classification theorem of connected components of the Fatou set (the complement of the Julia set) and from the fact that the boundaries of Siegel disks and Herman rings intersect the $\omega$-limit set of critical points (see [Bea and [CG for example) that if $f$ is not hyperbolic, then there must be a parabolic point in the Julia set. On the other hand, the existence of such a point obviously rules out hyperbolicity. Thus, we obtain the following.

Theorem 3.2. A rational function $f: J(f) \rightarrow J(f)$ is expansive but not hyperbolic if and only if the Julia set $J(f)$ contains no critical points of $f$ but contains at least one parabolic point. 
All expansive but not hyperbolic rational functions are called parabolic.

Since the Julia set of an expansive rational function $f: J(f) \rightarrow J(f)$ contains no critical points, also in this case we may define the function

$$
\mathrm{P}(t)=\mathrm{P}\left(f,-t \log \left|f^{\prime}\right|\right), t \in \mathbb{R},
$$

which was called the pressure function in the previous section. The basic properties of this function are collected in the following theorem. We include the statements for hyperbolic maps known from the previous section in order to emphasize the differences between the parabolic and hyperbolic cases.

Theorem 3.3. Suppose that $f: J(f) \rightarrow J(f)$ is expansive. Then

(a) The pressure function is convex and therefore continuous.

(b) The pressure function is non-increasing.

(c) If the mapping $f$ is hyperbolic, then the pressure function is strictly decreasing and $\lim _{t \rightarrow+\infty} \mathrm{P}(t)=-\infty$.

(d) If the mapping $f$ is parabolic, then there exists a number $s(f)>0$ such that $\mathrm{P}(t)=0$ for all $t \geq s(f)$ and $\left.\mathrm{P}\right|_{[0, s(f)]}$ is strictly decreasing.

Part (a) of this theorem is classical; the parts concerning parabolic maps ((b) and (d)) have been proved in DU3]. The proof makes extensive use of the variational principle and the non-negativity of Lyapunov exponents. The fact that in the parabolic case for all $t, \mathrm{P}(t) \geq 0$ is caused by the existence of invariant probability measures supported on (periodic) orbits of parabolic points. In the case of hyperbolic maps we denote the only zero of $\mathrm{P}(t)$ also by $s(f)$. The following theorem, which is classical in the hyperbolic case (see Section 2) and called the Bowen-Manning-McCluskey formula (see [Bo1]; cf. [MM]), has been proved in the parabolic case in DU3]. It can be considered as a strengthening of Theorem 1.9 and partial answer to the Problem 1.12. Since the function $t \mapsto \mathrm{P}(t)$ is real-analytic on the interval $[0, s(f))$ (see $\mathrm{By}$ ] and $\underline{\mathrm{Sm}}$ ), item (d) of Theorem 3.3 implies that at point $s(f)$ the real-analyticity of the pressure function breaks down. This phenomenon is called phase transition.

Theorem 3.4. If a rational function $f: J(f) \rightarrow J(f)$ is expansive, then we have the following equalities:

$$
\mathrm{DD}(J(f))=\mathrm{hD}(J(f))=\delta(f)=s(f)=\mathrm{HD}(J(f)) .
$$

Using Theorem 1.7 and the variational principle, one has no difficulty seeing that $s(f) \leq \mathrm{DD}(J(f))$. So, a priori, the largest number in Theorem 3.4 is $\operatorname{HD}(J(f))$, the Hausdorff dimension of the Julia set $J(f)$. We would like to provide the sketch of the proof that $\operatorname{HD}(J(f)) \leq \delta(f)$. In order to do this and later on to be able to discuss the so called uniform formula for conformal measure, we need the concept of hyperbolic zoom which appeared in [U2], which was extensively used in the author's papers with M. Denker and which got its name in [SU1].

From now on assume that the rational map $f: \overline{\mathbb{C}} \rightarrow \overline{\mathbb{C}}$ is parabolic. As will be seen in the sequel, parabolic maps form the class of rational functions formally strikingly similar to the class of geometrically finite Kleinian groups. The analogy between these two classes of rational functions and Kleinian groups takes on the clearest and most far-reaching form, providing, to our taste, one of the most beautiful chapters of Sullivan's dictionary.

We continue with the remark that in the parabolic case the $\omega$-limit set of critical points is contained in forward orbits of parabolic points and attracting periodic 
points. Therefore, given $e>0$, an expansive constant of $f: J(f) \rightarrow J(f)$, there exists a positive constant $\theta>0$ such that for every $z \in J(f) \backslash B(\Omega, e)$ the ball $B(z, \theta)$ is disjoint from $\mathrm{PCV}(f)$. Consequently all continuous inverse branches of all iterates $f^{n}, n \geq 1$, are well-defined on all the balls $B(z, \theta)$, where $z \in J(f) \backslash B(\Omega, e)$. It now follows from expansiveness of $f: J(f) \rightarrow J(f)$ that if $x \in J(f) \backslash \bigcup_{n \geq 0} f^{-n}(\Omega)$, then there exists an infinite increasing sequence $n_{j}(x), j \geq 1$, such that for every $j \geq 1$ we have

$$
f^{n_{j}(x)}(x) \notin B(\Omega, e) .
$$

We let $\left\{n_{j}(x)\right\}$ denote the maximal sequence satisfying the above property. Consequently we get the following:

Theorem 3.5. If $f: \overline{\mathbb{C}} \rightarrow \overline{\mathbb{C}}$ is a parabolic rational function, then the set of conical points $J_{c}(f)$ coincides with the set $J(f) \backslash \bigcup_{n \geq 0} f^{-n}(\Omega)$.

Exactly the same statement holds for geometrically finite Kleinian groups.

Theorem 3.5 and a fairly complete understanding of the dynamics of $f$ around parabolic points provide most basic tools for the theory of parabolic maps and play an important role in its further development. The sequence of numbers

$$
r_{j}(x)=\left|\left(f^{n_{j}(x)}\right)^{\prime}(x)\right|^{-1}
$$

is called the hyperbolic zoom at the point $x$. Applying Koebe's Distortion Theorem and the conformality property, one may easily observe that (cf. (1.2)) if $m$ is a $t$ conformal measure for $f$, then there exists a constant $B(m) \geq 1$ such that for all $x \in J_{c}(f)$

$$
B(m)^{-1} \leq \frac{m\left(B\left(x, r_{j}(x)\right)\right)}{r_{j}(x)^{t}} \leq B(m)
$$

We are now in a position to provide the proof of the following taken from [U2] (cf. [DU3]).

Theorem 3.6. If $\mathrm{H}_{t}$ is the t-dimensional Hausdorff measure on $J(f)$ and $m$ is a t-conformal measure for $f: J(f) \rightarrow J(f)$, then $\mathrm{H}_{t}$ is absolutely continuous with respect to $m$ such that the Radon-Nikodym derivative is bounded from above. Consequently, $t \geq \operatorname{HD}(J(f))$ and there is no t-conformal measure for $t<\operatorname{HD}(J(f))$.

Proof. Let $F \subset J(f)$ be any Borel set. Put $E=J_{c}(f) \cap F=F \backslash \bigcup_{n=0}^{\infty} f^{-n}(\Omega)$. Since the set $\bigcup_{n=0}^{\infty} f^{-n}(\Omega)$ is at most countable, $\mathrm{H}_{t}(E)=\mathrm{H}_{t}(F)$. Fix $\gamma, \epsilon>0$. Since $m$ is regular we can find a countable cover $\left\{B\left(x_{i}, r\left(x_{i}\right)\right)\right\}_{i=1}^{\infty}$ of $E$ of multiplicity bounded by a universal constant $C \geq 1$ (coming from the Besicovic covering theorem) such that $x_{i} \in E, 0<r\left(x_{i}\right)<\gamma$ is of the form $r_{j}\left(x_{i}\right)$ for every $i=1,2, \ldots$ (defined in (3.2)) and such that

$$
m\left(\bigcup_{i=1}^{\infty} B\left(x_{i}, r\left(x_{i}\right)\right) \backslash E\right)<\epsilon .
$$

Hence, applying (3.3) to the measure $m$, we obtain

$$
\begin{aligned}
\sum_{i=1}^{\infty} r\left(x_{i}\right)^{t} & \leq B(m) \sum_{i=1}^{\infty} m\left(B\left(x_{i}, r\left(x_{i}\right)\right)\right) \\
& \leq B(m) C m\left(\bigcup_{i=1}^{\infty} B\left(x_{i}, r\left(x_{i}\right)\right)\right) \leq C B(m)(\epsilon+m(E)) .
\end{aligned}
$$


Letting $\varepsilon \searrow 0$ and then $\gamma \searrow 0$, we get $\mathrm{H}_{t}(F)=\mathrm{H}_{t}(E) \leq C B(m) m(E) \leq$ $C B(m) m(F)$. The proof is complete.

As already announced, it follows immediately from this theorem that $\operatorname{HD}(J(f)) \leq$ $\delta(f)$. Thus the proof of Theorem 3.4 is complete.

Theorem 3.4 implies in particular that there are no $t$-conformal measures with exponents $t<\operatorname{HD}(J(f))$. The following theorem describes the structure of all conformal measures which has the same form as in the case of Kleinian groups.

Theorem 3.7. If $f: \overline{\mathbb{C}} \rightarrow \overline{\mathbb{C}}$ is a parabolic map, then the following three statements hold:

(a) There is no t-conformal measure with $t<\mathrm{HD}(J(f))$.

(b) There exists exactly one $\mathrm{HD}(J(f))$-conformal measure. This measure is atomless.

(c) For every $t>\operatorname{HD}(J(f))$ there are $t$-conformal measures. Each such measure is a convex combination of purely atomic measures supported on $\bigcup_{n \geq 0} f^{-n}(\Omega)$.

Thus, the structure of the set of all conformal measures in this (in a sense closest to the hyperbolic) case is substantially much more complex than in the hyperbolic case. As was already mentioned, item (a) of this theorem is already contained in Theorem 3.4. The existence of $\operatorname{HD}(J(f))$-conformal measures is also given by Theorem 3.4. The existence of an atomless $\operatorname{HD}(J(f))$-conformal measure is proved in Theorem 8.7 of $\mathrm{ADU}$ and its uniqueness is demonstrated in Theorem 4.6 of DU4. Finally, item (c) is proved in Theorem 13 of DU3.

Recently (see Section 3 and especially Section 5) there has been very strong interest in the Hausdorff dimension of various Julia sets. Particularly popular is the problem of whether the Hausdorff dimension of a Julia set is equal to 2 or less than 2. To our knowledge the first result in this direction which goes beyond the hyperbolic case has been obtained in $\mathrm{ADU}$, where the following theorem has been proved.

Theorem 3.8. If $f: \overline{\mathbb{C}} \rightarrow \overline{\mathbb{C}}$ is a parabolic map, then $\operatorname{HD}(J(f))<2$.

Below we provide the proof of this theorem. We give this proof (cf. the proof of Theorem 2.5) since the method employed there has turned out to be applicable also in other settings.

Proof of Theorem 3.8. First, using the concept of hyperbolic zooms, the distortion property on the balls $B\left(x, r_{j}(x)\right)$ and Lebesgue's density theorem, one demonstrates as in [Ly1] and [DH1] (cf. also [DU3] and the proof of Theorem 2.5) that the (2dimensional) Lebesgue measure of the Julia set $J(f)$ is equal to 0 . We now follow the proof of Theorem 8.8 in [ADU]. Suppose on the contrary that $\operatorname{HD}(J(f))=2$ and let $m$ be a 2-conformal measure existing by Theorem 3.4. For every $x \in J_{c}(f)$ let $\left\{r_{j}(x)\right\}_{j=1}^{\infty}$ be the hyperbolic zoom at the point $x$ introduced by (3.2). Fix $\varepsilon>0$ and denote by $\lambda$ the (2-dimensional) Lebesgue measure on $\mathbb{C}$. Since $\lambda(J(f))=0$ and since $\lim _{j \rightarrow \infty} r_{j}(x)=0$, for every $x \in J_{c}$ there exists a radius $r(x)$ being of the form $r_{j}(x)$ such that

$$
\lambda\left(\bigcup_{x \in J_{c}} B(x, r(x))\right)<\varepsilon .
$$

Now by the Besicovic covering theorem (see [Gu]; cf. [PU1]) we can choose a countable subcover $\left\{B\left(x_{i}, r\left(x_{i}\right)\right)\right\}_{i=1}^{\infty}$ of the cover $\{B(x, r(x))\}_{x \in J_{c}(f)}$ of $J_{c}(f)$, of multiplicity bounded by some universal constant $C \geq 1$. Therefore, using (3.3) with 
$t=2$, we obtain

$$
\begin{aligned}
m\left(J_{c}\right) & \leq \sum_{i=1}^{\infty} m\left(B\left(x_{i}, r\left(x_{i}\right)\right)\right) \leq B(m) \sum_{i=1}^{\infty} r\left(x_{i}\right)^{2}=B \pi^{-1} \sum_{i=1}^{\infty} \lambda\left(B\left(x_{i}, r\left(x_{i}\right)\right)\right) \\
& \leq B \pi^{-1} C \lambda\left(\bigcup_{i=1}^{\infty} B\left(x_{i}, r\left(x_{i}\right)\right)\right) \leq B C \pi^{-1} \varepsilon .
\end{aligned}
$$

Thus, letting $\varepsilon \rightarrow 0$, we obtain $m\left(J_{c}\right)=0$. Since, by Theorem $3.7(\mathrm{~b}), m$ is atomless, it implies that $m(J(f))=0$. This contradiction finishes the proof.

There is another nice way, not using conformal measures, to show that $\operatorname{HD}(J(f))$ $<2$ for parabolic maps, which shows even more that the upper box-counting dimension of these Julia sets is less than 2. Namely, a bounded subset $X$ of a Euclidean space is said to be porous if there exists a positive constant $c>0$ such that each open ball centered at a point of $X$ and of an arbitrary radius $0<r \leq 1$ contains an open ball of radius $c r$ disjoint from $X$. The notion of porosity has appeared in several contexts, and for a short survey and some bibliographical references the reader may see the paper Mat. By a simple counting argument it is straightforward to prove the following.

Theorem 3.9. If $X$ is a porous subset of $R^{d}$, then $\overline{\mathrm{BD}}(X)<d$.

L. Geyer has proved in Ge that

Theorem 3.10. The Julia set of a parabolic rational function is porous.

These two theorems imply the following.

Theorem 3.11. The upper box-counting dimension of the Julia set of a parabolic rational function is less than 2.

Notice however that in [DU5], using the jump transformation (see below), we have proved the following.

Theorem 3.12. If $f$ is a parabolic mapping, then $\overline{\operatorname{BD}}(J(f))=\operatorname{HD}(J(f))$.

To our surprise the proof of this theorem, similarly as its counterpart in the theory of Kleinian groups (see SU3]), is much more involved than in the hyperbolic case (see Theorem 2.4). In particular combining this theorem and Theorem 3.8, we get Theorem 3.11 .

For parabolic maps our interests went deeper to study the finer structure of Julia sets. We were interested in which cases Hausdorff and packing measures are finite and positive. The complete answer is given by the the following theorem, proven in DU6].

Theorem 3.13. If $f$ is a parabolic mapping and $h:=\operatorname{HD}(J(f))$, then

(a) $\mathrm{H}_{h}(J(f))<\infty$ and $\Pi_{h}(J(f))>0$.

(b) $\mathrm{H}_{h}(J(f))=0$ if and only if $h<1$.

(c) $\Pi_{h}(J(T))=\infty$ if and only if $h>1$.

It is really striking that the same result is true for Kleinian groups.

The proof of this theorem makes extensive use of the $h$-conformal measure $m$. The fact $\mathrm{H}_{h}(J(f))<\infty$ follows immediately from the first part of Theorem 3.6; the fact $\Pi_{h}(J(f))>0$ can be proved similarly. The results $\mathrm{H}_{h}(J(f))=0$ if $h<1$ and 
$\Pi_{h}(J(T))=\infty$ if $h>1$ are not too difficult, and their proofs use closer and closer visits of forward iterates of points from $J_{c}$ to $\Omega$. The hardest parts are to prove that $\mathrm{H}_{h}(J(f))>0$ if $h \geq 1$ and $\Pi_{h}(J(f))<\infty$ if $h \leq 1$. To see this we need to have a closer look at the ratio $\frac{m(B(x, r))}{r^{h}}$, where $m$ is the unique $h$-conformal measure. The first rather implicit description of this ratio has been made in [DU6], more explicit and extensive in [DU5], and eventually in [SU1] it took on the following final form closely resembling its counterpart in the theory of geometrically finite Kleinian groups.

Theorem 3.14. Let $f$ be a parabolic rational map with Julia set $J(T)$ of Hausdorff dimension $h$. Let $m$ denote the associated $h$-conformal measure supported on $J(T)$. Then there exists a function $\phi: J(f) \times \mathbb{R}^{+} \rightarrow \mathbb{R}^{+}$such that for each $z \in J(f)$ and for every positive $r<\operatorname{diam}(J(f))$ we have that

$$
m(B(z, r)) \asymp r^{h} \cdot \phi(z, r) .
$$

The function $\phi$ is called the conformal fluctuation function. For $r<\operatorname{diam}(J(f))$, the values of $\phi$ are determined as follows. For $z \in J_{c}(f)$, assume that $r$ relates to the hyperbolic zoom at $z$ such that $r_{j+1}(z) \leq r<r_{j}(z)$ and such that $f^{k}(z) \in B(\omega, e)$, for all $k \in\left(n_{j}(z), n_{j+1}(z)\right]$ and for some $\omega \in \Omega$. In this situation we have that

$$
\phi(z, r) \asymp \begin{cases}\left(\frac{r}{r_{j}(z)}\right)^{(h-1) p(\omega)} & \text { for } r \geq r_{j}(z)\left(\frac{r_{j+1}(z)}{r_{j}(z)}\right)^{1 /(1+p(\omega))} \\ \left(\frac{r_{j+1}(z)}{r}\right)^{(h-1)} & \text { for } r \leq r_{j}(z)\left(\frac{r_{j+1}(z)}{r_{j}(z)}\right)^{1 /(1+p(\omega))}\end{cases}
$$

If $z \in J_{p}$, then for every $r>0$ small enough

$$
\phi(z, r) \asymp r^{(h-1) p(\omega)} .
$$

Let

$$
p_{\max }=\max \{p(\omega): \omega \in \Omega\} .
$$

From now on till the end of this section we would like to present several further results specific for the parabolic case. They have no counterparts in the theory of hyperbolic systems, and their proofs are by no means modifications of the proofs in the hyperbolic case. They are specific for parabolic maps also because passing to the next class (NCP maps) most of these results (Theorems 3.15-3.21) are not known yet (most likely do not hold in such a complete form as in the parabolic case) and others have only partial analogs in the class of NCP maps. The third reason we include Theorems 3.15-3.21 in our survey is that they have exact counterparts in the theory of geometrically finite Kleinian groups ([St1], St2, [Su6]) and definitively demonstrate that both classes, parabolic rational functions and geometrically finite Kleinian groups, correspond to each other.

A finer analysis of the conformal fluctuation function $\phi$ done in [SU1] leads to the following theorem proven in [SU1] as Corollary 4.4.

Theorem 3.15. For the conformal fluctuation function $\phi$ of the h-conformal measure $m$ associated to a parabolic rational map $f$ the following holds.

(a) If $h=1$, then for all $z \in J_{c}(f)$ and all $0<r<\operatorname{diam}(J(T))$

$$
\phi(z, r) \asymp 1 .
$$


(b) If $h<1$, then for $m$-almost every $z \in J(f)$

$$
\limsup _{r \rightarrow 0} \frac{\log \phi(z, r)}{\log \log \frac{1}{r}}=\frac{(1-h) p_{\max }}{h+(h-1) p_{\max }} .
$$

(c) If $h>1$, then for $m$-almost every $z \in J(f)$

$$
\liminf _{r \rightarrow 0} \frac{\log \phi(z, r)}{\log \log \frac{1}{r}}=\frac{(1-h) p_{\max }}{h+(h-1) p_{\max }} .
$$

The main ingredient in the proof of this result was so called Khintchin Limit Law for parabolic rational maps proven in [SU1] (Theorem 4.3) with the help of the jump transformation and invariant measures absolutely continuous with respect to conformal measure, which will be discussed more extensively later in this section. This Khintchin law reads as follows.

Theorem 3.16. (The Khintchin Limit Law for parabolic rational maps) The hyperbolic zoom at $m$-almost every $z \in J(f)$ has the property that

$$
\limsup _{j \rightarrow \infty} \frac{\log \left(r_{j}(z) / r_{j+1}(z)\right)}{\log \log \frac{1}{r_{j}(z)}}=\frac{1+p_{\max }}{h+(h-1) p_{\max }} .
$$

Note that this Khintchin law is the analog of the logarithmic law of geodesics for limit sets of Kleinian groups (see $[\mathrm{SV}]$ ). Given $\rho \in \mathbb{R}$ let

$$
\psi_{\rho}(r):=r^{h}\left(\log \frac{1}{r}\right)^{(1+\rho)(1-h) p_{\max } /\left(h+(h-1) p_{\max }\right)}
$$

be a gauge function and let $\mathrm{H}_{\psi_{\rho}}$ and $\Pi_{\psi_{\rho}}$ be the corresponding Hausdorff and packing measure. As an immediate consequence of our finer analysis of the conformal fluctuation function (Theorem 3.15) we get the following result proven in SU1] (Corollary 4.5).

Theorem 3.17. Let $f$ be a parabolic rational map with Julia set $J(f)$ of Hausdorff dimension $h$ and with $h$-conformal measure $m$. We then have

(a) If $\rho>0$ and $h<1$, then $m<<\mathrm{H}_{\psi_{\rho}}$ and $\mathrm{H}_{\psi_{\rho}}(J(f))=\infty$.

(b) If $\rho>0$ and $h>1$, then there exists a Borel set $E_{\rho}$ such that $m\left(E_{\rho}\right)=1$ and $\Pi_{\psi_{\rho}}\left(E_{\rho}\right)=0$.

(c) If $\rho \leq 0$ and $h<1$, then there exists a Borel set $F_{\rho}$ such that $m\left(F_{\rho}\right)=1$ and $\mathrm{H}_{\psi_{\rho}}\left(F_{\rho}\right)=0$.

(d) If $\rho \leq 0$ and $h>1$, then $m<<\Pi_{\psi_{\rho}}$ and $\Pi_{\psi_{\rho}}(J(f))=\infty$.

We would like to remark that for $h<1$ similar results were obtained by different methods in DR.

Another direction of exploring the finer fractal structure of parabolic Julia sets is motivated by multifractal analysis and Diophantine approximation (see [Bes] and Ja ) of irrational numbers and the limit sets of Kleinian groups (see an extended discussion of this motivation in [SU2]). In order to define the setting briefly let us consider an expansive rational function $f: J(f) \rightarrow J(f)$ and a Hölder potential $g: J(f) \rightarrow \mathbb{R}$ satisfying $g \geq \log \left|f^{\prime}\right|$. Fix $x \in J(f)$. Following [HV] we then define 
the set

$$
\begin{aligned}
\mathcal{J}_{g, x}(f)=\{z \in J(f): & z \in B\left(y, \exp \left(-\sum_{j=0}^{n-1} g \circ f^{j}(y)\right)\right) \\
& \text { for infinitely many pairs } \left.(y, n) \text { such that } f^{n}(y)=x\right\} .
\end{aligned}
$$

In $[\mathrm{HV}]$ Hill and Velani have proved the following.

Theorem 3.18. If $f: \overline{\mathbb{C}} \rightarrow \overline{\mathbb{C}}$ is a hyperbolic rational function, then for every $x \in J(f), \operatorname{HD}\left(\mathcal{J}_{g, x}(f)\right)=s(g)$, where $s(g)$ is the only zero of the pressure function $t \mapsto \mathrm{P}(-t g)$.

It turns out that in the parabolic case the situation is much more complicated. In [SU2] we have considered the function $g=(1+\sigma) \log \left|f^{\prime}\right|,(\sigma \geq 0)$ and we have proved the following, where $\mathcal{J}_{x}^{\sigma}(f)=\mathcal{J}_{(1+\sigma) \log \left|f^{\prime}\right|, x}(f)$.

Theorem 3.19. Suppose that $f: \overline{\mathbb{C}} \rightarrow \overline{\mathbb{C}}$ is a parabolic rational function. Fix an element $x \in f^{-1}(\Omega) \backslash \Omega$. Then

(a) If $h<1$, then $\operatorname{HD}\left(\mathcal{J}_{x}^{\sigma}(f)\right)=\frac{h}{1+\sigma}$.

(b) If $h \geq 1$, then

$$
\operatorname{HD}\left(\mathcal{J}_{x}^{\sigma}(f)\right)= \begin{cases}\frac{h}{1+\sigma} & \text { for } \sigma \geq h-1 \\ \frac{h+\sigma p(\omega)}{1+\sigma(1+p(\omega))} & \text { for } \sigma<h-1 .\end{cases}
$$

Thus in the case when $h>1$ we observe again the phenomenon of a phase transition.

Given $\omega \in \Omega, y \in f^{-n}\left(f^{-1}(\omega) \backslash\{\omega\}\right)$ for some $n \geq 1$ and $\alpha>0$, we define $r(y)=\left|\left(f^{n}\right)^{\prime}(y)\right|^{-1}$ and $r_{y, \alpha}=\alpha^{1 /(1+p(\omega))} r(y)^{p(\omega) /(1+p(\omega))}$. Our approach heavily relied on the following Dirichlet cover type result and developed the methods worked out originally to tackle similar problems in the theory of Kleinian groups (see for example [St1] and [St2]).

Theorem 3.20. Suppose that $f: \overline{\mathbb{C}} \rightarrow \overline{\mathbb{C}}$ is a parabolic rational function. Fix an element $\omega \in \Omega$. Then there exist universal constants $\kappa_{c}, \kappa_{p}, \alpha_{0}>0$, depending only on $f$, such that for each $\omega \in \Omega$ and for each $0<\alpha<\alpha_{0}$ the following holds.

(a) The family $\left\{B\left(y, \kappa_{p} r_{y, \alpha}\right): r(y) \geq \alpha\right\}$ provides a packing of $J(f)$.

(b) The family $\left\{B\left(y, \kappa_{c} r_{y, \alpha}\right): r(y) \geq \alpha\right\}$ provides a covering of $J(f)$.

A simplified and clarified proof of Theorem 3.19 not using Theorem 3.20 was given in [SU4] in the context of finite parabolic iterated function systems.

We would like to draw the reader's attention especially to the case $h>1$ and $\sigma<h-1$ in Theorem 3.19, since it shows that the situation in the parabolic setting is much more complex than in the hyperbolic case and the most natural extension of Theorem 3.18 (requiring now $s(g)$ to be the least zero of the pressure function $\mathrm{P}(-t g))$ to the parabolic case fails since, due to Theorem 3.4, $s\left((1+\sigma) \log \left|f^{\prime}\right|\right)=$ $\frac{h}{1+\sigma}$.

The last part of this section is devoted to a discussion of the ergodic theory of an $h$-conformal measure $m$. This theory has been developed in [DU4] and ADU]. Recall (see $\left[\mathrm{Ru}, 7.29\right.$, p. 146]) that a family $\Re=\left\{R_{1}, \ldots, R_{s}\right\}$ is said to be a 
Markov partition for a continuous map $T: X \rightarrow X$ of a compact metric space $X$ if the following conditions are satisfied: $(\operatorname{Int}(A)$ will denote the interior of the set $A$ )

$$
\begin{aligned}
& R_{i}=\overline{\operatorname{Int}\left(R_{i}\right)} \quad \text { for every } R_{i} \in \Re . \\
& \text { If } i \neq j, \text { then } \operatorname{Int}\left(R_{i}\right) \cap \operatorname{Int}\left(R_{j}\right)=\emptyset .
\end{aligned}
$$

Each set $T\left(R_{i}\right)$ is a union of sets $R_{j} \in \Re$.

Denote by $\partial A$ the boundary of the set $A$. It was shown in Theorem 2.2 of [DU4] that each continuous expansive mapping of a compact metric space admits a metric compatible with the topology with respect to which this mapping is expanding. Since each open continuous expanding map admits Markov partitions of arbitrarily small diameters (see Theorem 2.4 and Lemma 2.3 from [DU4]; cf. [Ru] and [Bo2]) with boundaries of measure zero for a fixed atomless probability measure, we obtain the following.

Theorem 3.21. If $f: J(f) \rightarrow J(f)$ is a parabolic rational map and if $\mu$ is an atomless probability measure on $J(f)$, then $f: J(f) \rightarrow J(f)$ admits Markov partitions $\Re=\left\{R_{1}, \ldots, R_{s}\right\}$ of arbitrarily small diameters such that $\mu\left(\partial R_{1} \cup \ldots \cup \partial R_{s}\right)=0$.

Having a Markov partition $\Re$ with a sufficiently small diameter, define the associated jump transformation as follows. Denote by $R(\Omega)$ the union of all elements of $\Re$ that have non-empty intersection with $\Omega$. Given $z \in J(f) \backslash \Omega$ we define $n(z) \geq 0$ to be the least integer $n \geq 0$ such that $f^{n}(z) \notin R(\Omega)$. Define then the jump transformation $f^{*}: J(f) \backslash \Omega \rightarrow J(f)$ (depending on the Markov partition) by setting

$$
f^{*}(z)=f^{n(z)+1}(z) .
$$

The idea of the jump transformation is taken from [Sc1] (cf. also [Sc2]). Using this mapping and adapting the Schweiger formalism developed in [Sc1] to our context, we proved in [DU4] the following two theorems.

Theorem 3.22. If $f: J(f) \rightarrow J(f)$ is a parabolic rational map and $m$ is the only h-conformal measure, then for every jump transformation $f^{*}$ there exists a unique probability $f^{*}$-invariant measure $\mu^{*}$ absolutely continuous with respect to $m$. Moreover $\mu^{*}$ is ergodic and equivalent to $m$.

A $\sigma$-finite $f$-invariant measure $\nu$ is called conservative if for every set $A$ of positive measure

$$
\nu\left(\left\{x: \sum_{n \geq 0} \nu\left(T^{n}(x)\right)<\infty\right\}\right)=0 .
$$

Of course each finite $f$-invariant measure is conservative.

Theorem 3.23. If $f: J(f) \rightarrow J(f)$ is a parabolic rational map and $m$ is the only $h$-conformal measure, then there exists a, unique up to a multiplicative constant, $\sigma$-finite $f$-invariant measure $\mu$ absolutely continuous with respect to $m$. Moreover $\mu$ is ergodic, conservative and equivalent to $m$.

The concept of Schweiger's formalism was developed further in ADU] under the name of Markov fibered systems. We also heavily relied on Theorem 3.22 and additional properties of the jump transformation in [Sc1].

Definition 3.24. Suppose that $\mu$ is a $\sigma$-finite measure on a metric space $X$. We call $x \in X$ a point of infinite condensation of $\mu$ if and only if $\mu(U)=\infty$ for every open neighbourhood $\mu$ of $x$. Otherwise we call $x$ a point of finite condensation. 
In order to see at least one point of infinite condensation look at a sphere considered as the one-point (Alexandrov) compactification of a Euclidean space. This added point, usually denoted by $\infty$, is a point of infinite condensation of the Euclidean Lebesgue measure on this sphere.

In [DU4] we prove as Theorem 4.9 the following.

Theorem 3.25. If $f: J(f) \rightarrow J(f)$ is a parabolic rational map, then the set of points of infinite condensation of the invariant measure $\mu$ produced in Theorem 3.23 is contained in $\Omega$.

In [ADU] we have dealt with the problem of when the $\sigma$-finite invariant measure $\mu$ is finite. We gave there in Theorem 9.8 the following complete answer to this question.

Theorem 3.26. If $f: J(f) \rightarrow J(f)$ is a parabolic rational map, then the $\sigma$-finite invariant measure $\mu$ is finite if and only if

$$
h=\mathrm{HD}(J(f))>2 \max \left\{\frac{p(\omega)}{p(\omega)+1}\right\} .
$$

More precisely, we have the following.

Theorem 3.27. If $f: J(f) \rightarrow J(f)$ is a parabolic rational map and $\omega \in \Omega$, then $\mu$ is of infinite condensation at $\omega$ if and only if $h \leq \frac{2 p(\omega)}{p(\omega)+1}$.

Of course these last two theorems have no counterparts in the context of hyperbolic systems. As an immediate consequence of Theorem 3.26 we get the following.

Corollary 3.28. If $h=\mathrm{HD}(J(f)) \leq 1$, then the measure $\mu$ is infinite.

Corollary 3.29. If $h>1$ and $p(\omega)=1$ for every $\omega \in \Omega$, then $\mu$ is finite.

Observe that if $B: \overline{\mathbb{C}} \rightarrow \overline{\mathbb{C}}$ is a Blaschke product, then $J(B) \subset S^{1}$. So, as an immediate consequence of Corollary 3.28 we get the following.

Corollary 3.30. If $B: \overline{\mathbb{C}} \rightarrow \overline{\mathbb{C}}$ is a parabolic Blaschke product, then the $\sigma$-finite invariant measure $\mu$ is infinite.

Since it is proven (see [U2 and $[\mathrm{Zd}]$ ) that the Hausdorff dimension of the Julia set of the quadratic polynomial $z \mapsto z^{2}+1 / 4$ is greater than 1 , as an immediate consequence of Corollary 3.29 we get the following.

Corollary 3.31. The $\sigma$-finite invariant measure $\mu$ for the quadratic polynomial $z \mapsto z^{2}+1 / 4$ is finite.

Using the concept of equilibrium states for topological pressure (see Section 2) it turns out that Theorem 3.26 can be completed as follows.

Theorem 3.32. If $f: J(f) \rightarrow J(f)$ is a parabolic rational map, then the $\sigma$-finite invariant measure $\mu$ is finite if and only if one of the following two conditions holds.

(a) The potential $g=-h \log \left|f^{\prime}\right|$ has at least one atomless equilibrium state.

(b) The topological pressure function $t \mapsto \mathrm{P}(t)$ is not differentiable at the point $t=h$.

Thus, if the $\sigma$-finite measure $\mu$ is finite, then the pressure function has at the point $t=h$ a "stronger" phase transition than that observed in Theorem 3.3(b). 
We would like to conclude this section with the remark that recently parabolic bifurcations have been extensively investigated and lots of interesting dimension results have been obtained (see for ex. BB], [Mc1, [Sh], DSZ, [HS], UZ1], UZ2], [Zi1] and [Zi2]).

\section{Non-RECURREnt RAtional FunCtions (ALlowing PARABOliC Points)}

We recall that if $T: X \rightarrow X$ is a continuous map of a topological space $X$, then for every point $x \in X$, the $\omega$-limit set of $x$ denoted by $\omega(x)$ is defined to be the set of all limit points of the sequence $\left\{T^{n}(x)\right\}_{n \geq 0}$. We call a point $x$ recurrent if $x \in \omega(x)$; otherwise $x$ is called non-recurrent.

Definition 4.1. A rational function $f: \overline{\mathbb{C}} \rightarrow \overline{\mathbb{C}}$ is called a non-recurrent (NCP) map if all critical points of $f$ contained in the Julia set $J(f)$ are non-recurrent.

The class of NCP maps obviously contains all expanding as well as parabolic maps. It also comprises the important class of so called subexpanding maps which are defined by the requirement that $\left.f\right|_{\omega(\operatorname{Crit}(f)) \cap J(f)}$ is expanding and the class of geometrically finite maps defined by the property that the forward trajectory of each critical point contained in the Julia set is finite and disjoint from $\Omega$. The class of subexpanding maps was explored in [DU7] from the point of view of the fractal properties of the Julia set. We have dealt with NCP maps in [U3] and [U4] exploring their fractal and ergodic properties. Their geometric properties have been studied in CJY], and the rigidity type results have been obtained in [PU2]. In [Mc1 the reader may find some properties of geometrically finite maps proven earlier in [U3] for NCP maps. The paper $\mathrm{Ha}$ contains some ergodic properties of geometrically finite maps.

Since usually devastating behavior of critical points is relatively mild in the NCP case, the class of NCP maps shares lots of properties with the class of hyperbolic maps (if $\Omega(F)=\emptyset$ ) and with the class of parabolic maps (if $\Omega(f) \neq \emptyset$ ). Due to the lack of the existence of Markov partitions and the lack of a good analogue of the jump transformation, another method must be employed in order to explore NCP maps. The main points of this method consist of Marco Martens' approach to the problem of of the existence of $\sigma$-finite invariant measures (see Theorem 4.13), due to the fact (see Proposition 6.1 in [U3]) that all the points in $J(f) \backslash \bigcup_{n \geq 0} f^{-n}(\operatorname{Crit}(f) \cup$ $\Omega$ ) are conical and of an appropriate partial ordering of critical points of $f$ along with an appropriate stratification of the Julia set (see U3]). This last point plays an important role in the proof of the harder part of Theorem $4.6\left(\mathrm{H}_{h}(J(f))>\right.$ $\left.0, \Pi_{h}(J(f))<\infty\right)$. We would like to add that it is not clear whether there exists a satisfactory subclass of Kleinian groups analogous to the class of NCP maps.

Let us start our description of fractal and ergodic properties of NCP maps with the following characterization of conical points obtained in [U] as Proposition 6.1.

Theorem 4.2. If $f: \overline{\mathbb{C}} \rightarrow \overline{\mathbb{C}}$ is an NCP map, then

$$
J_{c}(f)=\overline{\mathbb{C}} \backslash \bigcup_{n \geq 0} f^{-n}(\Omega(f) \cup \operatorname{Crit}(J(f))) .
$$

As a sort of complementary result to this theorem we would like to state the following rather straightforward consequence of topological exactness of all rational functions restricted to their Julia sets, which was stated in [U3] (Lemma 5.2).

Theorem 4.3. The set $\omega(\operatorname{Crit}(J(f)))$ is nowhere dense in $J(f)$. 
Let, as before, $h=\mathrm{HD}(J(f))$ be the Hausdorff dimension of the Julia set $J(f)$. We proved in [U3] that there exists an $h$-conformal measure $m$ and we provided an incorrect argument that this measure is atomless (remaining essentially in the circle of methods worked out in [U3], it can be proved though that $m$ is atomless if $h \geq 1$ or if $f(\overline{\mathrm{PCV}(f)})$ is expanding). This affected some other statements included in [U3] and [U4]. Here we provide correct formulations. As Lemma 7.3 in U3] we have proved there the following.

Theorem 4.4. If the h-conformal measure $m$ is atomless, then $m$ is a unique $h$ conformal measure on $J(f)$ and $m$ is supported on the set of transitive points, i.e. those points whose $\omega$-limit set is the whole Julia set. If $m$ is not atomless, then it is supported on $\bigcup_{n \geq 0} f^{-n}(\operatorname{Crit}(J(f)))$.

In Proposition 7.4 of [U3] it is shown that Theorem 3.6 remains true for NCP maps. This, the existence of an $h$-conformal measure, and Theorem 1.9 immediately imply the following partial answer to Problem 1.12.

Theorem 4.5. If $f: J(f) \rightarrow J(f)$ is an $\mathrm{NCP}$ rational function, then we have the following equalities:

$$
\mathrm{DD}(J(f))=\mathrm{hD}(J(f))=\delta(f)=s(f)=\mathrm{HD}(J(f)) .
$$

Concerning geometric measures on the Julia sets of NCP maps, we have the following.

Theorem 4.6. Suppose that $f: \overline{\mathbb{C}} \rightarrow \overline{\mathbb{C}}$ is an NCP rational function. Then

(a) $\mathrm{H}_{h}(J(f))<\infty$, and if the $h$-conformal measure $m$ is atomless, then $\Pi_{h}(J(f))>0$.

(b) If $h \geq 1$, then $\mathrm{H}_{h}(J(f))>0$. In particular the $h$-conformal measure $m$ is atomless.

(c) If $h<1$ and $\Omega(f) \neq \emptyset$, then $\mathrm{H}_{h}(J(f))=0$.

(d) If $h \leq 1$ and the $h$-conformal measure $m$ is atomless, then $\Pi_{h}(J(f))<\infty$.

(e) If $h>1$ and $\Omega(f) \neq \emptyset$, then $\Pi_{h}(J(f))=\infty$.

Since it is straightforward to check that both Hausdorff and packing measures appearing in the above theorem are conformal when finite and positive, it follows from this theorem and uniqueness of $h$-conformal measure that the conformal measure $m$ has the geometrical characterization as a normalized Hausdorff measure if $h \geq 1$ or (if atomless) as a normalized packing measure if $h \leq 1$. Dealing still with geometry, let me add the following two results.

Theorem 4.7. If $f: \overline{\mathbb{C}} \rightarrow \overline{\mathbb{C}}$ is an $\mathrm{NCP}$ rational function, then either $J(f)=\overline{\mathbb{C}}$ or $\operatorname{HD}(J(f))<2$.

Theorem 4.8. If $f: \overline{\mathbb{C}} \rightarrow \overline{\mathbb{C}}$ is an $\mathrm{NCP}$ rational function such that $J(f) \neq \overline{\mathbb{C}}$, then the Julia set $J(f)$ is porous and consequently the upper box dimension $\overline{\mathrm{BD}}(J(f))<$ 2 .

The first of these two theorems has been stated in [U3], and the second one is proved in [PU3]. Let me add that in [U4] we have provided a number of sufficient conditions for $\operatorname{HD}(J(f))=\overline{\mathrm{BD}}(J(f))$; one of them is that $\operatorname{HD}(J(f)) \leq 1$. The proof of Theorem 4.8 relies on the following three results, which, besides the tools mentioned in the beginning of this section, belong to the basic toolbox for the class of NCP maps and Collet-Eckmann type maps (see Definition 5.6). 
Theorem 4.9. There exist $\theta>0$ and $M \geq 0$ such that for every $x \in J(f) \backslash$ $B(\Omega, \delta)$, every integer $n \geq 0$, every connected component $V$ of $f^{-n}(B(x, \theta))$ is simply connected and the restriction $\left.f^{n}\right|_{V}$ has at most $M$ critical points (counted with multiplicities).

Theorem 4.10. For each $\varepsilon>0$ and $D<\infty$ there are constants $C_{1}$ and $C_{2}$ such that the following holds for all rational maps $F: \overline{\mathbb{C}} \rightarrow \overline{\mathbb{C}}$, all $x \in \overline{\mathbb{C}}$, all $1 / 2 \leq r<1$ and all $0<\gamma \leq 1 / 2$ :

Assume that $V$ (resp. $\left.V^{\prime}\right)$ is a simply connected component of $F^{-1}(B(x, \gamma))$ (resp. $F^{-1}(B(x, \gamma / 2))$ with $V t V^{\prime} \mapsto V \subset V^{\prime}$. Assume further that $\overline{\mathbb{C}} \backslash V$ has diameter at least $\varepsilon$ and $F$ has at most $D$ critical points (counted with multiplicity) in $V$. Then

$$
\left|F^{\prime}(y)\right| \operatorname{diam}\left(V^{\prime}\right) \leq C_{1}(1-r)^{-C_{2}} \delta
$$

for all $y \in V^{\prime}$. Furthermore, if $r=1 / 2$ and $0<\tau<1 / 2$, let $B^{\prime \prime}=B(z, \tau \gamma)$ be any disk contained in $B(x, \gamma / 2)$ and let $V^{\prime \prime}$ be a component of $F^{-1}\left(B^{\prime \prime}\right)$ contained in $V^{\prime}$. Then

$$
\operatorname{diam}\left(V^{\prime \prime}\right) \leq C_{3} \operatorname{diam}\left(V^{\prime}\right)
$$

with $C_{3}=C_{3}(\tau, \varepsilon, D)$ and $\lim _{\tau \rightarrow 0} C_{3}(\tau, \varepsilon, D)=0$, and

(c) $\quad V^{\prime \prime}$ contains a disk of radius greater than or equal to $C_{4} \operatorname{diam}\left(V^{\prime}\right)$

around every preimage of $F^{-1}(z)$ that is contained in $V^{\prime \prime}$. Here $C_{4}=C_{4}(\tau, \varepsilon, D)>$ 0 .

Theorem 4.11. If $f: \overline{\mathbb{C}} \rightarrow \overline{\mathbb{C}}$ is a rational function and if $\Omega \neq \emptyset$, then for every $\rho>0$ there exists $c=c(\rho)>0$ such that for each $x \in J(f)$ and each $r$ such that $\rho \operatorname{dist}(x, \Omega) \leq r \leq 1$ there exists an open ball $B \subset B(z, r) \backslash J(f)$ with radius $c r$.

Theorem 4.9 follows from [Ma2, Theorem II]. See also [CJY] Theorem 2.1] or [U3. Lemma 2.12 and Lemma 5.1] where this theorem or its variants have been proved and substantially used. A crucial step in the proof of Theorem 4.9 is that given an arbitrary $\varepsilon>0$ there exists $\theta$ so that the diameters of all the above components are less than $\varepsilon$. Theorem 4.10 is a part of the "bounded distortion" lemma that has been proved in [Pr3, Lemma 2.1]. Finally Theorem 4.11 is a straightforward consequence of Fatou's flower theorem.

We would like to start now a discussion of ergodic theory of NCP maps. It will be based on [U4] and [Ha]. The starting point is the following result proved in [U4] as Theorem 4.2.

Theorem 4.12. If $f: \overline{\mathbb{C}} \rightarrow \overline{\mathbb{C}}$ is an $\mathrm{NCP}$ rational function and the $h$-conformal measure $m$ is atomless (in particular if $h \geq 1$ ), then up to a multiplicative constant there exists exactly one $f$-invariant $\sigma$-finite measure $\mu$ absolutely continuous with respect to $h$-conformal measure $m$. Moreover $\mu$ is equivalent to $m$, conservative and ergodic.

Since NCP maps lack Markov partitions and a good jump transformation, the idea of the proof of Theorem 4.12 is to apply a general, sufficient condition for the existence of $\sigma$-finite absolutely continuous invariant measure proven in [Mar. In order to formulate this condition suppose that $X$ is a $\sigma$-compact metric space, $m$ is a Borel probability measure on $X$, positive on open sets, and that a measurable map $T: X \rightarrow X$ is given with respect to which measure $m$ is quasi-invariant, 
i.e. $m \circ T^{-1}<<m$. Moreover we assume the existence of a countable partition $\alpha=\left\{A_{n}: n \geq 0\right\}$ of subsets of $X$ which are all $\sigma$-compact and of positive measure $m$. We also assume that $m\left(X \backslash \bigcup_{n \geq 0} A_{n}\right)=0$. If in addition, for all $m, n \geq 1$ there exists $k \geq 0$ such that

$$
m\left(T^{-k}\left(A_{m}\right) \cap A_{n}\right)>0,
$$

then the partition $\alpha$ is called irreducible. Martens' result comprising Proposition 2.7 and Theorem 2.2 of $\mathrm{Mar}$ reads as follows.

Theorem 4.13. Suppose that $\alpha=\left\{A_{n}: n \geq 0\right\}$ is an irreducible partition for $T: X \rightarrow X$. Suppose that $T$ is conservative and ergodic with respect to the measure $m$. If for every $n \geq 1$ there exists $K_{n} \geq 1$ such that for all $k \geq 0$ and all Borel subsets $A$ of $A_{n}$

$$
K_{n}^{-1} \frac{m(A)}{m\left(A_{n}\right)} \leq \frac{m\left(T^{-k}(A)\right)}{m\left(T^{-k}\left(A_{n}\right)\right)} \leq K_{n} \frac{m(A)}{m\left(A_{n}\right)},
$$

then $T$ has a $\sigma$-finite $T$-invariant measure $\mu$ absolutely continuous with respect to $m$. Additionally $\mu$ is equivalent to $m$, conservative and ergodic, and unique up to a multiplicative constant.

Proof of Theorem 4.12. Let us first sketch the proof of Theorem 4.13 given in [Mar] and then, as its consequence, a short proof of Theorem 4.12 taken from U4. One fixes an element $A \in \alpha=\left\{A_{n}: n \geq 0\right\}$ and then one considers the following sequences of measures:

$$
S_{k}(m)=\sum_{i=0}^{k-1} m \circ f^{-i} \quad \text { and } \quad Q_{k}(m)=\frac{S_{k}(m)}{S_{k}(m)(A)} .
$$

It is proven in $\mathrm{Mar}$ that each weak limit $\mu$ of the sequence $Q_{k}(m)$ (a sequence $\left\{\nu_{k}: k \geq 1\right\}$ of measures on $X$ is said to converge weakly if for all $n \geq 1$ the measures $\nu_{k}$ converge weakly on all compact subsets of $A_{n}$ ) is a required invariant measure and

$$
\mu(F)=\lim _{n \rightarrow \infty} Q_{k}(m)(F)
$$

for every Borel set $F \subset X$.

In order to complete the proof of Theorem 4.12 we only need to construct an irreducible partition $\alpha$ with property (4.1). Indeed, set $Y=J(f) \backslash(\mathrm{PCV} \cup \Omega)$. For every $y \in Y$ consider a ball $B(y, r(y))$ such that $r(y)>0, m(\partial B(y, r(y)))=0$, and $r(y)<(1 / 2) \operatorname{dist}(y, \mathrm{PCV} \cup \Omega)$. The balls $B(y, r(y)), y \in Y$, cover $Y$, and since $Y$ is a metric separable space, one can choose a countable cover, say $\left\{\tilde{A}_{n}: n \geq 0\right\}$, from them. We may additionally require that the family $\left\{\tilde{A}_{n}: n \geq 0\right\}$ is locally finite, that is that each point $x \in Y$ has an open neighborhood intersecting only finitely many balls $\tilde{A}_{n}, n \geq 0$. We now define the family $\alpha=\left\{A_{n}: n \geq 0\right\}$ inductively setting

$$
A_{0}=\tilde{A}_{0} \quad \text { and } \quad A_{n+1}=\tilde{A}_{n+1} \backslash \bigcup_{k=1}^{n} \overline{\tilde{A}_{n}}
$$

(and throwing away empty sets). Obviously $\alpha$ is a disjoint family and

$$
\bigcup_{n \geq 1} A_{n} \supset J(f) \backslash(\mathrm{PCV} \cup \Omega) \backslash \bigcup_{n \geq 0} \partial \tilde{A}_{n}
$$


whence in view of [U3, Corollary 7.2], $m\left(\bigcup_{n>0} A_{n}\right)=1$. The distortion condition (4.1) follows now from Koebe's distortion theorem with all constants $K_{n}=K$; the constant corresponding to the scale $1 / 2$ in this theorem and irreducibility of the partition $\alpha$ follow from openness of the sets $A_{n}$ and topological exactness of the map $f: J(f) \rightarrow J(f)$.

Assuming that the conformal measure $m$ is atomless (for instance assuming that $h \geq 1$ or that $f(\overline{\mathrm{PCV}(f)})$ is expanding), the following results describing the structure of the invariant measure $\mu$ have been proved in [U4]. These are not so complete as in the parabolic (and hyperbolic) case.

Theorem 4.14. If $\Omega=\emptyset$, then there exists an $f$-invariant probability measure $\mu$ equivalent to $\mathrm{m}$.

Theorem 4.15. If $\omega(\operatorname{Crit}(f)) \cap \Omega=\emptyset$, then the set of points of infinite condensation of $\mu$ is contained in $\Omega$.

Theorem 4.16. If $h=\mathrm{HD}(J(f)) \geq 1$, then the set of points of infinite condensation of $\mu$ is contained in the set of parabolic points.

Theorem 4.17. If $\omega \in \Omega \backslash \mathrm{PCV}(f)$, then $\mu$ is of infinite condensation at $\omega$ if and only if $h \leq \frac{2 p(\omega)}{p(\omega)+1}$.

Theorem 4.18. If $\omega \in \Omega$ and $h \leq \frac{2 p(\omega)}{p(\omega)+1}$, then $\mu$ has infinite condensation at $\omega$.

Theorem 4.19. If $c \in J(f)$ is a critical point of $f$ of order $q, \omega=f(c) \in \Omega$, and $h \leq \frac{2 q p(\omega)}{p(\omega)+1}$, then $\omega$ is of infinite condensation of measure $\mu$.

We would like to conclude this section with the result which is a combined consequence of Theorem 4.15 and Theorem 4.17 and which has been later proved as Theorem 1.2 in $\mathrm{Ha}$.

Theorem 4.20. If $f: \overline{\mathbb{C}} \rightarrow \overline{\mathbb{C}}$ is geometrically finite, then the invariant measure $\mu$ is finite if and only if $h>2 \max \left\{\frac{p(\omega)}{p(\omega)+1}: \omega \in \Omega\right\}$.

\section{OTHER MAPS}

The celebrated result of Bishop and Jones (see [BJ]) saying that the Hausdorff dimension of the limit set of a finitely generated but not geometrically finite Kleinian group is 2 has stimulated an extensive activity aiming to explain the situation for rational functions of the Riemann sphere $\overline{\mathbb{C}}$. It has been known (see Theorem 3.8 and Theorem 4.7) that the Hausdorff dimension of Julia sets $(\neq \overline{\mathbb{C}})$ of parabolic and NCP maps is less than 2. To the other pole of the spectrum belongs the following result of Shishikura [Sh] (see also [HS], [Zi2], and [Mc1]).

Theorem 5.1. $\sup \left\{\operatorname{HD}\left(J\left(f_{c}\right)\right)\right\}=2$, where the supremum is taken over all parameters $c$ such that the quadratic map $f_{c}(z)=z^{2}+c$ is parabolic. The set of parameters $c$ such that $\operatorname{HD}\left(J\left(f_{c}\right)\right)=2$ is dense in the boundary of the Mandelbrot set.

The first result in the opposite direction, going beyond NCP maps, was due to F. Przytycki, who proved in [Pr4] the following.

Theorem 5.2. There exists a set $E \subset \mathbb{R}$ of positive Lebesgue measure such that $\operatorname{HD}\left(J\left(f_{c}\right)\right)<2$ for all $c \in E$. 
Since then a number of similar results have been obtained. Let us list some of them below.

Theorem 5.3. ([Mc2]) If $f(z)=e^{2 \pi i \theta} z+z^{2}$, where $\theta$ is a badly approximable irrational number (of bounded type) (so, $f$ has a Siegel disk) then the Julia set $J(f)$ is porous and consequently $\mathrm{HD}(J(f)) \leq \overline{\mathrm{BD}}(J(f))<2$.

A quadratic map $f_{c}(z)=z^{2}+c$ is a Fibonacci map if, loosely speaking, the closest returns of the critical point 0 occur precisely at the moments given by Fibonacci sequence $S_{n}=S_{n-1}+S_{n-2}$ and $S_{0}=S_{1}=2$. Graczyk and Smirnov proved in GS1 the following.

Theorem 5.4. If $f_{c}: \overline{\mathbb{C}} \rightarrow \overline{\mathbb{C}}$ is a real $(c \in \mathbb{R})$ quadratic Fibonacci map, then $\operatorname{HD}\left(J\left(f_{c}\right)\right)<2$.

A simply connected domain $A \subset \overline{\mathbb{C}}$ is called a Hölder domain (with exponent $\alpha \in(0,1])$ if the Riemann mapping $R: D^{1} \rightarrow A\left(D^{1}=\{z \in \mathbb{C}:|z|<1\}\right)$ can be extended in a Hölder continuous fashion (with exponent $\alpha$ ) to the closed disk $\overline{D^{1}}$. P. Jones and N. Makarov proved in [JM] the following.

Theorem 5.5. There exists a constant $c>0$ such that for every $\alpha \in(0,1]$ and every Hölder domain with exponent $\alpha, \operatorname{HD}(\partial A) \leq 2-c \alpha$.

Definition 5.6. A rational function $f: \overline{\mathbb{C}} \rightarrow \overline{\mathbb{C}}$ is called Collet-Eckmann if there are constants $C>0$ and $\lambda>1$ such that for every critical point in $J(f)$ whose forward orbit does not contain any other critical point, and every $n \geq 1$

$$
\left|\left(f^{n}\right)^{\prime}(f(c))\right| \geq C \lambda^{n} .
$$

Graczyk and Smirnov proved in [GS2] the following.

Theorem 5.7. The basin of attraction to $\infty$ of any Collet-Eckmann polynomial is a Hölder domain. In particular, due to Theorem 5.5 the Hausdorff dimension of its Julia set is strictly less than 2.

In PR and PU3] some extensions of the last part of this theorem have been proved. In fact a weaker form of porosity has been established there sufficient to conclude that the upper box counting dimension is less than 2 .

Definition 5.8. A set $X \subset \mathbb{R}^{d}, d \geq 1$, is called mean porous if there are constant $p_{1}<\infty$ and $p_{2}>0$ such that for each $z \in E$ there exist an increasing sequence $n_{j}$ of positive integers and a sequence of points $z_{j} \in \mathbb{R}^{d}$ with $\left\|z-z_{j}\right\| \leq 2^{-n_{j}}$ such that $n_{j}<p_{1} j$ and and $\operatorname{dist}\left(z_{j}, X\right)>p_{2} 2^{-n_{j}}$.

In $[\mathrm{KR}$ the following extension of Theorem 3.9 has been proved.

Theorem 5.9. If $X$ is a mean porous subset of a Euclidean space $R^{d}$, then $\overline{\mathrm{BD}}(X)$ $<d$.

The main result of $[\mathrm{PR}]$ reads as follows.

Theorem 5.10. The Julia set of a Collet-Eckmann rational function with no parabolic points is mean porous. In particular, due to Theorem 5.9, its upper boxcounting dimension is strictly less than 2. 
In [PU3] an appropriate definition of Collet-Eckmann rational functions with parabolic points is provided (loosely speaking one requires (5.1) to take the form $\left|\left(f^{n}\right)^{\prime}(f(c))\right| \geq C \lambda^{\phi(n)}$ for an appropriately changed time $\left.\phi(n) \leq n\right)$ and the following theorem has been proved.

Theorem 5.11. The Julia set of a Collet-Eckmann rational function with parabolic points is mean porous. In particular, due to Theorem 5.9, its upper box-counting dimension is strictly less than 2.

Another direction of recent activity has been stimulated by Problem 1.12 (see also Theorem 1.9). In [Pr3] F. Przytycki has proved the following.

Theorem 5.12. If $f: \overline{\mathbb{C}} \rightarrow \overline{\mathbb{C}}$ is a Collet-Eckmann map (in fact it suffices here to assume that $\left.\left|\left(f^{n}\right)^{\prime}(f(c))\right| \geq C e^{\theta \sqrt{n}}\right)$, then $\mathrm{DD}(J(f))=\mathrm{HD}(J(f))$. If additionally $f$ has no parabolic points, then $\operatorname{HD}(J(f))=\overline{\mathrm{BD}(J(f))}$.

and

Theorem 5.13. If $f(z)=z^{2}+c$ is a non-renormalizable quadratic polynomial with $c$ in the complement of the main cardioid in the Mandelbrot set, then $\operatorname{DD}(J(f))=$ $\operatorname{HD}(J(f))$.

We would like to conclude this section with the remark that in the papers $[\operatorname{Pr} 3$, Pr4, [PR] and [PU3] so called Rule II (see [DPU]) has been used extensively. Its formulation and the context surrounding it may be found in Appendix 2.

\section{APPEndix 1. TAME FUnCTIONS}

Unless stated otherwise all the results formulated in this appendix are taken from [PU2]. As in the previous sections let $f: \overline{\mathbb{C}} \rightarrow \overline{\mathbb{C}}$ be a rational function of the Riemann sphere $\overline{\mathbb{C}}$. By $T(f)$ we denote the set of all points in $J(f)$ for which

$$
\limsup _{n \rightarrow \infty} \operatorname{dist}\left(f^{n}(z), \operatorname{PCV}(f)\right)>0 .
$$

Definition A1.1. We say that a rational function $f: \overline{\mathbb{C}} \rightarrow \overline{\mathbb{C}}$ is tame if $\operatorname{PCV}(f) \cap$ $J(f)$ is nowhere dense in $J(f)$ and there exists a $t$-conformal measure $m$ (sometimes, to be more specific, denoted by $\left.m_{f}\right)$ such that $m(T(f))=1$.

Remark A1.2. The set $T(f)$ was already considered in GPS (called there the transverse limit set). In fact its origin goes back to [Ly1.

Remark A1.3. Notice that if the singular set $\operatorname{PCV}(f)$ of an arbitrary rational function $f$ is nowhere dense in $J(f)$, then $T(f)$ contains the set of all transitive points of $f$ (i.e. points with dense forward orbit). Therefore for such a function to be tame it is sufficient to have a conformal measure $m$ supported on the set of transitive points.

Clearly $T(f)$ is a subset of the set of conical points of $f$, and therefore as an immediate consequence of Theorem 1.11 we get the following.

Theorem A1.4. If $f: \overline{\mathbb{C}} \rightarrow \overline{\mathbb{C}}$ is tame, then there exists at most one value $t$ for which a t-conformal measure exists and is supported on $T(f)$. Additionally, for such a t there exists exactly one $t$-conformal measure supported on $T(f)$ and this measure is ergodic. 
Thus the measure $m=m_{f}$ and the exponent $t=t_{f}$ are determined uniquely. It is easy to see that $m_{f}$ is non-atomic. We can say something more about the exponent $t=t_{f}$. Indeed, with considerations similar to those in the proof of Theorem 3.6, we can demonstrate the following.

Theorem A1.5. If a rational function $f: \overline{\mathbb{C}} \rightarrow \overline{\mathbb{C}}$ is tame, then the $t_{f}$-dimensional Hausdorff measure of $T(f)$ is finite. In particular $t_{f} \geq \operatorname{HD}(T(f))$.

Employing the method introduced by M. Martens in Mar and proceeding in the same way as in the proof of Theorem 4.2 in U2 (see Theorem 4.11 and the discussion surrounding it), we can demonstrate the following.

Theorem A1.6. If a rational function $f: \overline{\mathbb{C}} \rightarrow \overline{\mathbb{C}}$ is tame, then up to a multiplicative constant there exists exactly one $f$-invariant $\sigma$-finite measure $\mu=\mu_{f}$ absolutely continuous with respect to $m$. Moreover, $\mu$ is equivalent to $m$, conservative and ergodic.

Examples of tame functions are provided for instance by NCP maps. Indeed, Theorem 4.2 and Theorem 4.4 of this paper, along with the paragraph preceding Theorem 4.4, imply the following.

Theorem A1.7. Each NCP rational function $f$ with an atomless conformal measure is tame and $t_{f}=\mathrm{HD}(J(f))$. Moreover, the $t_{f}$-conformal measure is supported on the set of transitive points.

The main result of the paper [PU2] is the following.

Theorem A1.8. Suppose that $f$ and $g$ are two tame rational maps. Let $h$ be an invertible map from a full measure $m_{f}$ subset of $J(f)$ onto a full measure $m_{g}$ subset of $J(g)$, preserving the algebras of measurable sets for $m_{f}$ and $m_{g}$ and conjugating $f$ to $g$, namely $h \circ f=g \circ h$. Then the following conditions (1)-(6) are equivalent.

(1) $h$ extends to a Möbius conjugacy between $f: \overline{\mathbb{C}} \rightarrow \overline{\mathbb{C}}$ and $g: \overline{\mathbb{C}} \rightarrow \overline{\mathbb{C}}$.

(2) $h$ extends to a conformal homeomorphism conjugating $f$ and $g$ on neighborhoods of $J(f)$ and $J(g)$ in $\overline{\mathbb{C}}$.

(3) $h$ extends to a real-analytic diffeomorphism conjugating $f$ and $g$ on neighborhoods of $J(f)$ and $J(g)$ in $\overline{\mathbb{C}}$.

(4) $h$ extends to a homeomorphism from $J(f)$ to $J(g)$ such that $h$ and $h^{-1}$ are Lipschitz continuous.

(5) $h$ extends to a homeomorphism from $J(f)$ to $J(g)$ such that for every periodic point $x$ of $f$, say of period $p,\left|\left(f^{p}\right)^{\prime}(x)\right|=\left|\left(g^{p}\right)^{\prime}(h(x))\right|$.

(6) The measure class of $m_{f}$ is transported under $h$ to the measure class of $m_{g}$.

Here for the implication $(6) \Rightarrow(2), f$ and $g$ are assumed not to be critically finite with parabolic orbifold (see [Th Ch.13], [DH2, §9] or [Zd] for the definition) for which $J(f)=J(g)=\overline{\mathbb{C}}$. We call such maps exceptional.

Remark A1.9. The implication $(5) \Rightarrow(2)$ was in fact proved by E. Prado in Pra] for all rational maps. The only missing point, the non-linearity (see [PU2] for the definition) caused by parabolic points turns out to be easy. Prado's proof of the only hard part $(5) \Rightarrow(2)$ was done by approximating $J(f)$ by forward invariant expanding repellers inside $J(f)$, where $h$ extends conformally; see [Su3], [Pr5]. Our proof of the implication $(5) \Rightarrow(2)$ is different and goes via (6). This requires the assumption that $f$ is tame. Thus the main new result in our paper is the implication 
$(6) \Rightarrow(2)$ which extends Sullivan's result for non-linear repellers (see [Su3] and [Pr5]); we use the same scheme of proof. This implication is called rigidity. Note that the implication $(2) \Rightarrow(1)$ is straightforward. This holds even in a general, not just tame, situation. Any conformal conjugacy on neighborhoods of Julia sets for any two rational functions extends to a conformal conjugacy (Möbius map) to the whole sphere. In particular by Theorem A1.9 the measure theoretic conjugacy class of a tame map coincides with its conformal conjugacy class. In other words one cannot perturb a tame map inside the measure theoretic conjugacy class changing its conformal conjugacy class. Equivalence classes of measures classify tame maps.

A rational function $f: \overline{\mathbb{C}} \rightarrow \overline{\mathbb{C}}$ is said to be Julia real-analytic if its Julia set is contained in a finite union of pairwise disjoint real-analytic curves which will be denoted by $\Gamma=\Gamma_{f}$. Frequently in such a context one alternatively speaks about real analyticity of the Julia set $J(f)$. We conclude this section with two technical ingredients, interesting by themselves, which have been proved in PU2] as Corollary 2.4 and Proposition 2.3 respectively, the first being an immediate consequence of the second.

Theorem A1.10. If $f: \overline{\mathbb{C}} \rightarrow \overline{\mathbb{C}}$ is tame, then the Jacobian of the map $f: J(f) \rightarrow$ $J(f)$ with respect to the measure $\mu$ has a real-analytic extension on a neighbourhood of $J(f) \backslash \overline{\mathrm{PCV}(f)}$ in $\mathbb{C}$. If the map $f$ is Julia real-analytic, then the Jacobian has a real-analytic extension on a neighbourhood of $J(f) \backslash \mathrm{PCV}$ in $\Gamma$.

Theorem A1.11. If $f: \overline{\mathbb{C}} \rightarrow \overline{\mathbb{C}}$ is a tame mapping, then the Radon-Nikodym derivative $\rho=d \mu / d m$ has a real-analytic real-valued extension on a neighbourhood of $J(f) \backslash \overline{\mathrm{PCV}(f)}$ in $\mathbb{C}$. If $f$ is Julia real-analytic, then $\rho$ has a real-analytic extension on a neighbourhood of $J(f) \backslash \overline{\mathrm{PCV}(f)}$ in $\Gamma$.

\section{Appendix 2. Equilibrium states and Perron-Frobenius operator}

In this appendix $f: \overline{\mathbb{C}} \rightarrow \overline{\mathbb{C}}$ is a rational function of the Riemann sphere $\overline{\mathbb{C}}$ (no additional assumptions imposed) and $\phi: J(f) \rightarrow \mathbb{R}$ is a Hölder continuous function satisfying the following condition:

$$
\mathrm{P}(\phi)=\mathrm{P}(\phi, f)>\sup (\phi),
$$

where, let us recall, $\mathrm{P}(\phi)$ is topological pressure defined between Theorem 3.2 and Theorem 3.3. We start with the following general result of Lyubich (see [Ly2]).

Theorem A2.1. If $f: \overline{\mathbb{C}} \rightarrow \overline{\mathbb{C}}$ is a rational function, then the function $\mu \mapsto$ $h_{\mu}(f)$ is upper semi-continuous, and consequently each continuous potential has an equilibrium state.

In fact in Ly2 M. Lyubich proved more: namely that each rational function is asymptotically $h$-expansive, the notion introduced by M. Misiurewicz in $\mathrm{Mi}$ in connection with his investigations of continuous maps of an interval. Recall from DU1] that a normalized Borel measure $\nu$ on a Borel measure space $(X, \mathcal{F})$ is called $g$-conformal for the continuous mapping $T: X \rightarrow X$, where $g: X \rightarrow \mathbb{R}$ is a positive continuous function if $\nu(T(A))=\int_{A} g d \nu$ for every $A \in \mathcal{F}$ such that $T \mid A$ is invertible. The paper [DU1] contains some basic facts about this notion and, in particular, sufficient conditions for the existence of these measures. The central result of [DU8] is the following. 
Theorem A2.2. Let $f: \overline{\mathbb{C}} \rightarrow \overline{\mathbb{C}}$ be a rational function and let $\phi: \overline{\mathbb{C}} \rightarrow \mathbb{R}$ be a Hölder continuous function satisfying (A2.1). Then:

(a) There exists exactly one $\exp (c-\phi)$-conformal probability measure $m$ and necessarily $c=P(\phi)$.

(b) There exists exactly one equilibrium state $\mu$ for $\phi$ and $f$. The measures $\mu$ and $m$ are equivalent and $\rho=d \mu / d m$ is a positive continuous function.

(c) The dynamical system $(J(f), f, \mu)$ is metrically exact and, consequently, its natural extension is a K-system.

Let us add that later in [Pr6] F. Przytycki proved the same results and that in the special case of the measure of maximal entropy $(\phi=0)$ some partial results have been obtained in [FLM], Ly2, and [Ma3]. In order to formulate results concerning the Perron-Frobenius operator (to be defined later) let us recall that a bounded linear operator $A: X \rightarrow X$ on a complex Banach space $X$ is called almost periodic if for every $x \in X$ the family $\left\{A^{n}(x)\right\}_{n=1}^{\infty}$ is relatively compact in the strong topology. The basic property of almost periodic operators proven in Ly2 is the following.

Theorem A2.3. If a bounded linear operator $A: X \rightarrow X$ on a complex Banach space $X$ is almost periodic, then the Banach space $X$ decomposes into a direct sum $X_{u} \oplus X_{0}$ of two closed, A-invariant subspaces $X_{u}$ and $X_{0}$ such that

(a) $X_{u}$ is the closure of the linear span of the unitary eigenvectors of $A$. (An eigenvector is called unitary if its corresponding eigenvalue is of modulus 1.)

(b) $X_{0}=\left\{x \in X: \lim _{n \rightarrow \infty} A^{n}(x)=0\right\}$.

The Perron-Frobenius operator $\mathcal{L}: C(J(f)) \rightarrow C(J(f))$ is defined by the following formula:

$$
\mathcal{L}(g)(x)=\sum_{y \in f^{-1}(x)} g(y) \exp (\phi(y)-\mathrm{P}(\phi)),
$$

where every critical point $y \in f^{-1}(x)$ is counted with its multiplicity. The main result on spectral properties of the Perron-Frobenius operator is the following theorem proven in [DU8] as Theorem 35 and later in [Pr6] (see also [Ly2 for the special case $\phi=0)$.

Theorem A2.4. The Perron-Frobenius operator $\mathcal{L}: C(J(f)) \rightarrow C(J(f))$ is almost periodic. Let $\rho$ denote the Radon-Nikodym derivative of the equilibrium state $\mu$ for $\phi$ and $f$ with respect to the $\exp (\mathrm{P}(\phi)-\phi)$-conformal measure $m$. Then

$$
(C(J(f)))_{u}=\mathbb{C} \rho \text { and }(C(J(f)))_{0}=\left\{\phi: \int \phi d \nu=0\right\} .
$$

Moreover, if $g=g_{u}+g_{0}$ with $g_{u} \in(C(J(f)))_{u}$ and $g_{0} \in\left(C(J(f))_{0}\right.$, then $g_{u}=$ $\left(\int g d m\right) \rho$.

In [DPU] we have proved the following results improving spectral properties of the Perron-Frobenius operator and our knowledge about the Radon-Nikodym derivative $d \mu / d m$.

Theorem A2.5. Let $\phi: J(f) \rightarrow \mathbb{R}$ be a Hölder continuous potential. Then there are constants $L>0$ and $\theta>0$ such that

$$
\left\|\mathcal{L}^{n}(\phi)-\rho \int \phi d m\right\|_{\infty} \leq L \exp (-\theta \sqrt{n})
$$

for all $n \geq 0$. 
Theorem A2.6. The Radon-Nikodym derivative of the equilibrium measure $\mu$ of the potential $\phi$ with respect to the $\exp (P(\phi)-\phi)$-conformal measure $m$ is a Hölder continuous function.

Using Gordin's method (see Go]) as an immediate consequence of Theorem A2.5, we obtained in [DPU] the following.

Theorem A2.7. Every Hölder continuous function $g: J(f) \rightarrow \mathbb{R}$ satisfies the central limit theorem. Namely there exists $\sigma \geq 0$ such that

$$
\lim _{n \rightarrow \infty} \frac{1}{n} \int\left(\sum_{k=0}^{n-1}\left(g \circ f^{k}-\mu(g)\right)\right)^{2} d \mu=\sigma^{2},
$$

and if $\sigma^{2}>0$, then for any $t \in \mathbb{R}$

$$
\mu\left(\left\{z \in J(f): \frac{1}{\sqrt{n \sigma^{2}}} \sum_{k=0}^{n-1}\left(g\left(f^{k}(z)\right)-\mu(g)\right) \leq t\right\}\right) \rightarrow \frac{1}{\sqrt{2 \pi}} \int_{-\infty}^{t} \exp \left[-u^{2} / 2\right] d u .
$$

We would like to notice that in $\overline{\mathrm{DPU}}$ we have proved the result, called Rule II, which played an important role there and in later activity in the field, especially in the work of F. Przytycki (see [Pr3], [Pr4], [PR] and [PU3]). In order to state this result, define for every $c \in \overline{\mathbb{C}}$ the function $k_{c}: \overline{\mathbb{C}} \rightarrow\{0,1,2, \ldots\} \cup\{\infty\}$ by setting

$$
k_{c}(x)=\min \left\{n \geq 0: x \notin B\left(c, a e^{-(n+1)}\right)\right\}
$$

and $k_{c}(x)=\infty$ if $x=c$. Here $a$ is an arbitrary positive number such that $a<$ diam $\overline{\mathbb{C}}$. Rule II is the following.

Theorem A2.8. (Rule II). There exists a constant $Q>0$ such that if $c \in J(f)$ is a critical point of $f, n \geq 1$ is an integer, and if $x \in J(f)$ satisfies

$$
k_{c}\left(f^{j}(x)\right) \leq k_{c}\left(f^{n}(x)\right) \quad \text { for every } \quad j=1,2, \ldots, n-1,
$$

then

$$
\min \left\{k_{c}(x), k_{c}\left(f^{n}(x)\right)\right\}+\sum_{j=1}^{n-1} k_{c}\left(f^{j}(x)\right) \leq Q n .
$$

We would like to conclude the above considerations with the remark that recently N. Haydn has improved (see $[\mathrm{Hay}]$ ) Theorem A2.5 substantially by demonstrating the following.

Theorem A2.9. Let $\phi: J(f) \rightarrow \mathbb{R}$ be a Hölder continuous potential. Then there are constants $L>0$ and $\theta>0$ such that

$$
\left\|\mathcal{L}^{n}(\phi)-\rho \int \phi d m\right\|_{\infty} \leq L \exp (-\theta n)
$$

for all $n \geq 0$.

In Ma4 R. Mané has proved that a power of each dynamical system $\left(J(f), f, \mu_{0}\right)$ is isomorphic to a one-sided Bernoulli shift, where $\mu_{0}$ is the measure of maximal entropy. D. Heicklen and Ch. Hoffman have recently proved in $[\mathrm{HH}]$ the following improvement of Mané's result.

Theorem A2.10. Every dynamical system $\left(J(f), f, \mu_{0}\right)$ is isomorphic to a onesided Bernoulli shift, where $\mu_{0}$ is the measure of maximal entropy. 


\section{ACKNOWLEDGMENT}

The author is deeply grateful to Bernd Stratmann, who carefully read the first draft of this article and made many helpful comments and corrections. He also wishes to thank the referees and Professors Doug Lind and Donald G. Saari, whose helpful comments and suggestions improved considerably the final exposition of this article.

\section{REFERENCES}

[ADU] J. Aaronson, M. Denker, M. Urbański, Ergodic theory for Markov fibred systems and parabolic rational maps, Transactions of A.M.S. 337 (1993), 495-548. MR 94g:58116

[Bea] A.F. Beardon, Iteration of Rational Functions, Springer-Verlag, New York, 1991. MR 92j:30026

[Bes] A.S. Besicovitch, 'Sets of fractional dimension(IV): On rational approximation to real numbers', Jour. London Math. Soc. 9 (1934), 126-131.

[BJ] C. Bishop, P. Jones, Hausdorff dimension and Kleinian groups, Acta Math. 179 (1997), 1-39. MR 98k:22043

[BZ] O. Bodart, M. Zinsmeister, Quelques resultats sur la dimension de Hausdorff des ensembles polynomes quadratiques, Fund. Math. 151 (1996), 121-137. MR 97i:30034

[Bo1] R. Bowen, Hausdorff dimension of quasi-circles, Publ. Math. IHES, 50 (1979), 11-25. MR 81g:57023

[Bo2] R. Bowen, Equilibrium states and the ergodic theory for Anosov diffeomorphisms. Lect. Notes in Math. 470, Springer, 1975. MR 56:1364

[BS] R. Bowen, C. Series, Markov maps associated with Fuchsian groups, Publ. Math. IHES 50 (1979), 153-179. MR 81b:58026

[BK] M. Brin, A. Katok, On local entropy, in Geometric Dynamics, Lect. Notes in Math. 1007 (1983), 30-38, Springer Verlag. MR 85c:58063

[By] J. Byrne, Multifractal analysis of parabolic rational maps, Ph.D thesis, Univ. of North Texas (1998).

[Ca] L. Carleson, On the support of harmonic measure for sets of Cantor type, Ann. Acad. Sci. Fenn. 10 (1985), 113-123. MR 87b:31002

[CG] L. Carleson, T.W. Gamelin, Complex Dynamics, Springer-Verlag, New York, 1993. MR 94h:30033

[CJY] L. Carleson, P. W. Jones, J.-Ch. Yoccoz, Julia and John, Bol. Soc. Bras. Mat. 25 (1994), 1-30. MR 95d:30040

[DMNU] M. Denker, D. Mauldin, Z. Nitecki, M. Urbański, Conformal measures for rational functions revisited, Fundamenta Math. 157 (1998), 161-173. MR 99j:58122

[DPU] M. Denker, F. Przytycki, M. Urbański, On the transfer operator for rational functions on the Riemann sphere, Ergod. Th. and Dynam. Sys. 16 (1996), 255-266. MR 97e:58197

[DR] M. Denker, S. Rohde, On Hausdorff Measures and SBR Measures for Parabolic Rational Maps, Internat. J. Bifur. Chaos Appl. Sci. Engrg. 9 (1999), no. 9, 1763-1769. MR 2001k:37070

[DU1] M. Denker, M. Urbański, On the existence of conformal measures, Trans. A.M.S. 328 (1991), 563-587. MR 92k:58155

[DU2] M. Denker, M. Urbański, On Sullivan's conformal measures for rational maps of the Riemann sphere, Nonlinearity 4 (1991), 365 - 384. MR 92f:58097

[DU3] M. Denker, M. Urbański, Hausdorff and conformal measures on Julia sets with a rationally indifferent periodic point, J. London Math. Soc. 43 (1991), 107-118. MR 92k:58153

[DU4] M. Denker, M. Urbański, On absolutely continuous invariant measures for expansive rational maps with rationally indifferent periodic points, Forum Math. 3(1991), 561579. MR 92k:58154

[DU5] M. Denker, M. Urbański, The capacity of parabolic Julia sets, Math. Zeitsch. 211, (1992), 73-86. MR 93j:30022

[DU6] M. Denker, M. Urbański, Geometric measures for parabolic rational maps, Ergod. Th. and Dynam. Sys. 12 (1992), 53-66. MR 93d:58133 
[DU7] M. Denker, M. Urbański, On Hausdorff measures on Julia sets of subexpanding rational maps, Israel J. of Math. 76 (1991), 193-214. MR 93g:58078

[DU8] M. Denker, M. Urbański, Ergodic theory of Equilibrium states for rational maps, Nonlinearity 4 (1991), 103-134. MR 92a:58112

[DH1] A. Douady, J.H. Hubbard, Etude dynamique des polynomes complexes I,II, Publications mathematique d'Orsay 84-2, 1984; 85-4, 1985. MR 87f:58072a MR 87f:58072b

[DH2] A. Douady, J.H. Hubbard, A proof of Thurston's topological characterization of rational functions, Acta Math. 171.2 (1993), 263-297. MR 94j:58143

[DSZ] A. Douady, P. Sentenac, M. Zinsmeister, Implosion parabolique et dimension de Hausdorff, C. R. Acad. Sci, Paris, 325 (Serie 1) (1997), 765-772. MR 98i:58195

[Fa] K. Falconer, Fractal geometry, Mathematical Foundations and Applications, John Wiley \& Sons, 1990. MR 92j:28008

[FLM] A. Freire; A. Lopes; R. Mañé: An invariant measure for rational maps, Bol. Soc. Bras. Mat. 14 (1983), 45-62. MR 85m:58110b

[Ge] L. Geyer, Porosity of parabolic Julia sets, Complex Variables Theory Appl. 39 (1999), 191-198. MR 2000e:37055

[Go] M.I. Gordin, The central limit theorem for stationary processes. Dokl. Akad. Nauk SSSR 188 (1969), 1174-1176. MR 40:5012

[GS1] J. Graczyk, S. Smirnov, The Fibonacci Julia set, conformal measures and Hausdorff dimension, Preprint.

[GS2] J. Graczyk, S. Smirnov, Collet, Eckmann and Hölder, Invent. Math. 133 (1998), 69-96. MR 2000a:37029

[Gr] M. Gromov, On the entropy of holomorphic maps, Preprint IHES.

[GPS] P. Grzegorczyk, F. Przytycki, W. Szlenk, On iterations of Misiurewicz's rational maps on the Riemann sphere, Ann. Inst. Henri Poincaré, 53 (1990), 431-434. MR 92d:30017

[Gu] M. de Guzmán, Differentiation of integrals in $\mathbb{R}^{n}$. Lect. Notes in Math. 481, Springer Verlag, 1975. MR 56:15866

[HK] B. Hasselblatt, A. Katok, Introduction to the modern theory of dynamical systems, Encyclopedia of Mathematics and its Applications 54, Cambridge University Press, 1995. MR 96c:58055

[Ha] G. Havard, Mesures invariantes pour les fractions rationnelles geometriquement finies, Fund. Math. 160 (1999), 39-61. MR 2000h:37068

[Hay] N. Haydn, Convergence of the transfer operator for rational maps, Ergodic Theory Dynam. Systems 19 (1999), 657-669. MR 2000f:37055

$[\mathrm{HH}] \quad$ D. Heicklen, C. Hoffman, Rational maps are $d$-adic Bernoulli, Ann. of Math. (2) 156 (2002), 103-114.

[HS] S. Heinemann, B. Stratmann, Hausdorff dimension 2 for Julia sets of quadratic polynomials, Math. Z. 237 (2001), 571-583. MR 2002d:37079

[HV] R. Hill, S. Velani, Metric Diophantine approximation in Julia sets of expanding rational maps, Publ. Math. 85 (1997), 193-216. MR 99b:58143

[Ja] V. Jarník, Diophantische Approximationen and Hausdorff Mass, Mathematicheskii Sbornik 36 (1929), 371-382.

[JM] P. Jones, N. Makarov, Density properties of harmonic measure, Ann. of Math. 142 (1995), 427-455. MR 96k:30027

[KS] M. Kesseböhme, B. Stratmann, A multifractal analysis for growth rates and applications to geometrically finite Kleinian groups, Preprint 2001.

[KR] P. Koskela, S. Rohde, Hausdorff dimension and mean porosity, Math. Ann. 309 (1997), 593-609. MR 98k:28004

[Ly1] M. Lyubich, On a typical behaviour of trajectories for a rational map of sphere, Dokl. Ak. N. U.S.S.R. 268 (1982), 29-32. MR 84f:30036

[Ly2] M. Lyubich, Entropy properties of rational endomorphisms of the Riemann sphere. Ergod. Th. Dynam. Sys. 3 (1983), 351-386. MR 85k:58049

[Ma1] R. Mané, The Hausdorff dimension of invariant probabilities of rational maps, Dynamical Systems, Valparaiso 1986, Lect. Notes in Math. 1331, Springer-Verlag (1988), 86-117. MR 90j:58073

[Ma2] R. Mané, On a theorem of Fatou. Bol. Soc. Brasil. Mat. 24 (1993), 1-12. MR 94g:58188

[Ma3] R. Mané, On the uniqueness of the maximizing measure for rational maps, Bol. Soc. Bras. Mat. 14 (1983), 27-43. MR 85m:58110a 
[Ma4] R. Mané, On the Bernoulli property of rational maps. Ergod. Th. Dynam. Sys. 5 (1985), 71-88. MR 86i: 58082

$[\mathrm{MM}] \quad$ A. Manning, H. McCluskey, Hausdorff dimension for horseshoes, Ergod. Th. and Dynam. Sys. 3 (1983), 251-260. MR 85j:58127

[Man] A. Manning, The dimension of a maximal measure for a polynomial map, Ann. of Math. (2) 119 (1984), 425-430. MR 85i:58068

[Mar] M. Martens, The existence of $\sigma$-finite invariant measures, Applications to real onedimensional dynamics, Preprint SUNY Stony Brook IMS preprint 1992/1.

[Mat] P. Mattila, Geometry of sets and measures in euclidean spaces, Cambridge Studies in Advanced Mathematics 44, Cambridge University Press, 1995. MR 96h:28006

[May] V. Mayer, Private communication, 2001.

[Mc1] C. McMullen, Hausdorff dimension and conformal dynamics II: Geometrically finite rational maps, Comment. Math. Helv. 75 (2000), 535-593. MR 2001m:37089]

[Mc2] C. McMullen, Self-similarity of Siegel disks and Hausdorff dimension of Julia sets, Acta Math. 180 (1998), 247-292. MR 99f:58172

[Mc3] C. McMullen, Hausdorff dimension and conformal dynamics III: Computation of dimension, Amer. J. Math. 120 (1998), 691-721. MR 2000d:37055

[Mi] M. Misiurewicz, Topological conditional entropy. Studia Math. 55 (1976), 175-200. MR 54:3672

[Pa1] S. J. Patterson, The limit set of a Fuchsian group, Acta Math. 136 (1976), 241-273. MR 56:8841

[Pa2] S. J. Patterson, Lectures on measures on limit sets of Kleinian groups, in Analytical and geometric aspects of hyperbolic space, London Math. Soc., Lecture Notes 111, Cambridge Univ. Press, 1987. MR 89b:58122

[Pe] Ya. Pesin, Dimension theory in dynamical systems, University of Chicago Press (1997). MR 99b:58003

[PW] Ya. Pesin, H. Weiss, The multifractal analysis of Gibbs measures: Motivation, Mathematical foundation and examples, Chaos 7:1 (1997), 89-106. MR 98e:58130

[Pra] E. Prado, Teichmüller distance for some polynomial-like maps, SUNY Stony Brook IMS preprint 1996/2, revision 1997.

[Pr1] F. Przytycki, Lyapunov characteristic exponents are non-negative. Proc. Amer. Math. Soc. 119.1 (1993), 309-317. MR 93k:58193

[Pr2] F. Przytycki, Hausdorff dimension of harmonic measure on the boundary of an attractive basin for a holomorphic map, Invent. Math. 80 (1985), 169-171. MR 86g:30035

[Pr3] F. Przytycki, Iterations of holomorphic Collet-Eckmann maps: conformal and invariant measures. Trans. AMS 350.2 (1998), 717-742. MR 98d:58155

[Pr4] F. Przytycki, On measure and Hausdorff dimension of Julia sets for holomorphic ColletEckmann maps, International conference on dynamical systems, Montevideo 1995, Pitman Research Notes in Math. 362 (1996), 167-181. MR 98i:58198

[Pr5] F. Przytycki, Sullivan's classification of conformal expanding repellers, Preprint 1991, to appear in the book "Fractals in the plane - ergodic theory methods" by F. Przytycki and M. Urbański.

[Pr6] F. Przytycki, On the Perron-Frobenius-Ruelle operator for rational maps on the Riemann sphere and for Hölder continuous functions. Bol. Soc. Bras. Mat. 20 (1990), 95-125. MR 93b:58120

[PR] F. Przytycki and S. Rohde, Porosity of Collet-Eckmann Julia sets. Fund. Math. 155 (1998), 189-199. MR 2000b:37047

[PU1] F. Przytycki, M. Urbański, Fractals in the Plane - the Ergodic Theory Methods, available on the web:http://www.math.unt.edu/urbanski, to appear in Cambridge Univ. Press.

[PU2] F. Przytycki, M. Urbański, Rigidity of tame rational functions, Bull. Pol. Acad. Sci., Math., 47.2 (1999), 163-182. MR 2000i:37065

[PU3] F. Przytycki, M. Urbański, Porosity of Julia sets of non-recurrent and parabolic ColletEckmann rational functions, Ann. Acad. Fenn. 26 (2001), 125-154. MR 2002b:37063

[PUZ, I] F. Przytycki, M. Urbański, A. Zdunik, Harmonic, Gibbs and Hausdorff measures on repellers for holomorphic maps I, Ann. of Math. 130 (1989), 1-40. MR 91i:58115

[PUZ, II] F. Przytycki, M. Urbański, A. Zdunik, Harmonic, Gibbs and Hausdorff measures on repellers for holomorphic maps II, Studia Math. 97 (1991), 189-225. MR 93d:58140 
[RT] C. Rogers, S. Taylor, Functions continuous and singular with respect to a Hausdorff measure, Mathematika, 8 (1961), 1-31. MR 24:A200

[Ru] D. Ruelle, Thermodynamic formalism, Encyclopedia of Math. and Appl., vol. 5, Addison - Wesley, Reading, Mass., 1978. MR 80g:82017

[Sc1] F. Schweiger: Number theoretical endomorphisms with $\sigma$-finite invariant measures. Isr. J. Math. 21 (1975), 308-318. MR 52:5608

[Sc2] F. Schweiger, Ergodic Theory of Fibred Systems and Metric Number Theory, Oxford Sci. Publ., Oxford University Press, New York, 1995. MR 97h:11083

[Sh] M. Shishikura, The Hausdorff Dimension of the Boundary of the Mandelbrot Set and Julia Sets, Ann. of Math. 147 (1998), 225-267. MR 2000f:37056

[Si] C. L. Siegel, Iteration of analytic functions, Ann. of Math. 43 (1942), 607-612. MR 4:76c

[Sm] S. Smirnov, Spectral Analysis of Julia sets, Thesis (1996).

[St1] B. Stratmann, Fractal dimensions for Jarnik limit sets of geometrically finite Kleinian groups; the semi-classical approach, Ark. för Mat. 33 (1995), 385-403. MR 97a:30056

[St2] B. Stratmann, Weak singularity spectra of the Patterson measure for geometrically finite Kleinian groups with parabolic elements, Michigan Math. J. 46 (1999), 573-587. MR 2001a:37059

[SU1] B. O. Stratmann, M. Urbański, The Geometry of Conformal Measures for Parabolic Rational Maps, Math. Proc. Cambridge Phil. Soc. 128 (2000), 141-156. MR 2000i:37066

[SU2] B. O. Stratmann, M. Urbański, Jarnik and Julia; a Diophantine analysis for parabolic rational maps, Math. Scan. 91 (2002), 27-54.

[SU3] B. O. Stratmann, M. Urbański, The box-counting dimension for geometrically finite Kleinian groups, Fundamenta Mathematica 149 (1996), 83-93. MR 96m:30062

[SU4] B. O. Stratmann, M. Urbański, Metrical Diophantine analysis for tame parabolic iterated function systems, Preprint 2000.

[SV] B. O. Stratmann, S. Velani, The Patterson measure for geometrically finite groups with parabolic elements, new and old, Proc. London Math. Soc. 71 (1995) page 197-220. MR 97f:58023

[Su1] D. Sullivan, Seminar on conformal and hyperbolic geometry. Preprint IHES (1982).

[Su2] D. Sullivan, Conformal dynamical systems. In: Geometric dynamics, Lect. Notes in Math. 1007, Springer Verlag (1983), 725-752. MR 85m:58112

[Su3] D. Sullivan, Quasiconformal homeomorphisms in dynamics, topology, and geometry, Proc. Internat. Congress of Math., Berkeley, Amer. Math. Soc., 1987, 1216-1228. MR 90a:58160

[Su4] D. Sullivan, The density at infinity of a discrete group, Inst. Hautes Etudes Sci. Pub. Math. 50 (1979). MR 81b:58031

[Su5] D. Sullivan, Entropy, Hausdorff measures old and new, and limit sets of geometrically finite Kleinian groups, Acta. Math. 153 (1984), 259-277. MR 86c:58093

[Su6] D. Sullivan, Disjoint Spheres, Approximation by Imaginary Quadratic Numbers and the Logarithmic Law for Geodesics, Acta Math. 149 (1982) 215-237. MR 84j:58097

[TT] S. J. Taylor, C. Tricot, Packing measure, and its evaluation for a Brownian path, Trans. A.M.S. 288 (1985), 679-699. MR 87a:28002

[Th] W. Thurston, Three-Dimensional Geometry and Topology, Princeton University Press, 1997. MR 97m:57016

[U1] M. Urbański, On Some Aspects of Fractal Dimensions in Higher Dimensional Dynamics, in Proc. of the Göttingen Workshop Problems on Higher Dimensional Complex Dynamics, Mathematica Gottingensis 3 (1995) 18-25.

[U2] M. Urbański, On Hausdorff dimension of a Julia set with a rationally indifferent periodic point, Studia Math. 97 (1991), 167-188. MR 93a:58146

[U3] M. Urbański, Rational functions with no recurrent critical points, Ergod. Th. and Dynam. Sys. 14 (1994), 391-414. MR 95g:58191

[U4] M. Urbański, Geometry and ergodic theory of conformal nonrecurrent dynamics, Ergod. Th. and Dynam. Sys. 17 (1997), 1449-1476. MR 99j:58178

[UZ] M. Urbański, A. Zdunik, Hausdorff dimension of harmonic measure for self-conformal sets, Adv. Math. 171 (2002), 1-58.

[UZ1] M. Urbański, M. Zinsmeister, Geometry of hyperbolic Julia-Lavaurs sets, Indagationes Math. 12 (2001) 273 - 292. 
[UZ2] M. Urbański, M. Zinsmeister, Continuity of Hausdorff dimension of Julia-Lavaurs sets as a function of the phase, Journal of Conformal Geometry and Dynamics, 5 (2001), 140-152. MR 2002j:37058

[Vo] A. Volberg, On the dimension of harmonic measure of Cantor repellers, Mich. Math. J. 40 (1993), 239-258. MR 95d:30043

[Wa1] P. Walters, An introduction to ergodic theory, Springer-Verlag, 1982. MR 84e:28017

[Wa2] P. Walters, A variational principle for the pressure of continuous transformations, Amer. J. Math. 97 (1975), 937 - 971. MR 52:11006

[Zd] A. Zdunik, Parabolic orbifolds and the dimension of maximal measure for rational maps, Inv. Math. 99 (1990), 627-649. MR 90m:58120

[Zi1] M. Zinsmeister (after A. Douady), Basic parabolic implosions in five days, Preprint $1997 / 8$.

[Zi2] M. Zinsmeister, Fleur de Leau-Fatou et dimension de Hausdorff, C. R. Acad. Sci. Paris Sér. I Math. 326 (1998), 1227-1232. MR 99j:58180

Department of Mathematics, University of North Texas, P.O. Box 311430, Denton, TEXas 76203-1430

E-mail address: urbanski@unt.edu 